\title{
Plasticity versus specificity in RTK signalling modalities for distinct biological outcomes in motor neurons
}

Nathalie Caruso, Balazs Herberth, Fabienne Lamballe, Vilma Arce-Gorvel, Flavio Maina and Françoise Helmbacher ${ }^{*}$

\begin{abstract}
Background: Multiple growth factors are known to control several aspects of neuronal biology, consecutively acting as morphogens to diversify neuronal fates, as guidance cues for axonal growth, and as modulators of survival or death to regulate neuronal numbers. The multiplicity of neuronal types is permitted by the combinatorial usage of growth factor receptors, each of which is expressed in distinct and overlapping subsets of neurons, and by the multitasking role of growth factor receptors, which recruit multiple signalling cascades differentially required for distinct biological outcomes. We have explored signalling robustness in cells where a given receptor tyrosine kinase (RTK) elicits qualitatively distinct outcomes. As the HGF/Met system regulates several biological responses in motor neurons (MN) during neuromuscular development, we have investigated the signalling modalities through which the HGF/Met system impacts on MN biology, and the degree of robustness of each of these functions, when challenged with substitutions of signalling pathways.

Results: Using a set of mouse lines carrying signalling mutations that change the Met phosphotyrosine binding preferences, we have asked whether distinct functions of Met in several MN subtypes require specific signalling pathways, and to which extent signalling plasticity allows a pleiotropic system to exert distinct developmental outcomes. The differential ability of signalling mutants to promote muscle migration versus axonal growth allowed us to uncouple an indirect effect of HGF/Met signalling on nerve growth through the regulation of muscle size from a direct regulation of motor growth via the PI3 kinase (PI3K), but not Src kinase, pathway. Furthermore, we found that HGF/Met-triggered expansion of Pea3 expression domain in the spinal cord can be accomplished through several alternative signalling cascades, differentially sensitive to the Pea3 dosage. Finally, we show that the regulation of MN survival by HGF/Met can equally be achieved in vitro and in vivo by alternative signalling cascades involving either PI3K-Akt or Src and Mek pathways.
\end{abstract}

Conclusions: Our findings distinguish MN survival and fate specification, as RTK-triggered responses allowing substitutions of the downstream signalling routes, from nerve growth patterning, which depends on a selective, non-substitutable pathway.

Keywords: Motor neurons, RTK signalling, Survival, Cell fate specification, Development, HGF/Met

\footnotetext{
* Correspondence: Francoise.HELMBACHER@univ-amu.fr

Aix-Marseille Université, IBDM, CNRS UMR 7288, Parc Scientifique de Luminy,

Case 907, 13288 Marseille, France
} 


\section{Background}

The assembly of locomotor circuits relies on the parallel development of spinal motor neurons (MNs) and their target muscles, and subsequently, on their coordinated functional wiring. This involves the stepwise acquisition of cell diversification programs in both cell types, each of which leads to the generation of distinct subtypes, the subsequent matching of each motor pool with its cognate target muscle through topographical arrangement of their axonal projections, and finally a process that allows numerical matching of $\mathrm{MN}$ pool size to the size of the target muscle $[1,2]$. During these consecutive phases, a number of aspects of MN biology, including specification, axon growth, guidance and survival, are controlled by multiple target-derived growth factors and their receptors [1]. Most of these growth factors are capable of eliciting multiple responses and the specialisation appears to involve the subtype-specific distribution of their cognate receptors, respectively established through the combinatorial distribution of fate-specifying transcription factors [3]. The resulting combinations of growth factor receptors define a unique set of growth factor responses for each $\mathrm{MN}$ subtype, matching either specific muscles or a functional subtype $[4,5]$.

An additional level of complexity appears when considering signalling modalities. The actions of each growth factor receptor upon ligand binding involve the activation of cytoplasmic signalling pathways [6]. The discovery by Tony Pawson of conserved domains in signalling molecules conferring binding ability to activated receptor tyrosine kinases (RTKs) has revolutionised how we see that RTK signalling regulates cellular behaviours. In particular, a profusion of cytoplasmic signalling effectors, binding phosphorylated tyrosines via Src homology domain type 2 (SH2) and type 3 (SH3) and phosphotyrosine binding domain (PTB), can be recruited upon receptor activation by their ligands, to mediate multiple, qualitatively different, biological responses to a given factor. One signalling adaptor can be used as an effector of different biological responses to distinct growth factors. Conversely, a given growth factor receptor can employ distinct signalling effector pathways to mediate distinct cellular responses. Introducing point mutations in the genomic loci of RTKs, such as TrkC, TrkB, Met, Pdgfr or Ret, by selectively modifying the binding sites through which they recruit cytoplasmic effectors, has been instrumental in distinguishing the respective contribution of signalling adaptors, such as PLC $\gamma$, Shc, Grb2, PI3K and Src, in mediating qualitatively distinct cellular responses in vivo [7-12]. The studies cited above compared cellular behaviours (such as survival, migration, proliferation and target innervation) adopted by cell types with very different biological histories (i.e. neurons versus muscles or vascular smooth muscle cells) but did not distinguish distinct subtypes (molecular or anatomical) within a generic cell type. However, RTK signalling requirements for a given biological response might not always be identical in different cell types or subtypes. Signalling requirements may be determined by the intrinsic competence of each cell type to provide a robust signal transduction platform capable of executing the function, such competence being directly linked to the intrinsic molecular identity of the cell. Thus, to define the robustness of a specific biological outcome requires understanding for a cell type the degree to which several alternative signalling pathways can substitute for each other to execute qualitatively distinct RTK-driven responses, and distinguishing permissive from non-permissive pathways. We chose to address this issue by modulating the signalling competences of a growth factor/RTK system in charge of multiple biological functions in one unique cell type, focusing on MNs, and by exploring specificity versus plasticity of the pathways that intercalate to execute each of these biological responses.

Among the plethora of factors known to influence $\mathrm{MN}$ biology, we chose to focus on hepatocyte growth factor (HGF), a multifunctional growth factor whose functions are mediated by the Met RTK. HGF influences MN biology by acting both on MNs and on muscles. Produced by the limb bud mesenchyme, HGF first acts on muscle migration by triggering the delamination of myoblast precursors from limb level somites [13]. This event is necessary for myoblasts to undergo their long-range migration towards the limbs [13-15]. In embryos lacking Met signalling, the absence of limb muscles causes the death of all limb MNs $[4,16]$, most likely by depleting all musclederived neurotrophic factors required to support MN survival. Besides this indirect influence, HGF/Met signalling also plays a number of direct functions in MNs, including regulating motor axon growth $[4,9,17,18]$, MN subtype specification $[4,16]$ and MN survival $[4,18,19]$.

Using tissue-specific ablation of the Met receptor, we have recently shown that as development proceeds, HGF acts on distinct MN pools, in which it successively controls several specific biological pathways [4]. On a subset of Met-expressing brachial MNs, HGF acts at early stages (during a muscle-independent period), by influencing cell fate specification (through cell autonomous induction of expression of the transcription factor Runx1 and non-cell autonomous induction of the transcription factor Pea3 in neighbouring neurons) and consequently axon growth toward and within their target muscle (cutaneous maximus (CM)) [4,9,16]. After the onset of muscle-dependency for MN survival, highlighted by a peak of naturally occurring cell death (NOCD), which spreads among motor columns to eliminate supernumerary MNs [20], we recently discovered a switch in both the biological response and in the MN pools supported by HGF/Met. As shown with neural-specific ablation of Met 
after NOCD, HGF/Met signalling is required for the survival of a distinct pool of Met-expressing MNs that innervate the pectoralis minor muscle, whereas it is no longer required to support CM MNs [4]. Thus, the biological response to a given target-derived growth factor depends both on the MN subtype and on timing, offering an interesting context in which to address robustness versus specificity of signalling requirements.

In this study, we took advantage of an allelic series of met, including: a) the $m e t^{d}$ signalling dead allele [15]; b) the hypomorphic $m e t^{2 P}$ and $m e t^{2 S}$ signalling mutant alleles, in which the multifunctional docking site is converted into optimal binding sites for phosphatidylinositol 3-kinase (PI3K) or Src, respectively [9]; c) an alternative knockout/knock-in $m e t^{\text {LacZ }}$ allele [4] and d) a conditional $m e t^{\text {Flox }}$ allele $[4,21]$. We asked whether execution of three different biological responses (axonal patterning, specification and survival) elicited by the pleiotropic RTK Met in distinct MN subtypes, is compatible with substitutions of the signalling pathways, or requires selective non-substitutable signalling routes. By uncoupling Met functions in MNs from its function in muscle migration, and by selectively allowing Met to signal through either Src or PI3K optimal binding sites, we found that: 1) while HGF/Met signalling indirectly impacts on nerve growth through the regulation of muscle size in the limbs, a direct regulation of motor growth is achieved in the presence of limiting amounts of muscles by activating PI3K, but not Src, pathway; 2) the non-cell autonomous function of HGF/Met that allows expansion of the Pea3 expression domain in the spinal cord [16], can be accomplished through several alternative signalling cascades, although their competence is differentially sensitive to Pea3 dosage; 3) regulation of $\mathrm{MN}$ survival by $\mathrm{HGF} / \mathrm{Met}$ can be equally achieved in vitro and in vivo by alternative signalling cascades involving either PI3K-Akt or Src and Mek pathways.

\section{Results}

Most signalling by the Met receptor is mediated by two C-terminal tyrosines, referred to as multifunctional docking sites. The surrounding amino-acid environment ( $\mathrm{Y}_{1349} \mathrm{VHVNATY}_{1356} \mathrm{VNV}$ ) is permissive to the binding of multiple signalling adaptors (Figure 1A) [22]. Whereas converting both tyrosines to phenylalanine completely abrogates signal transduction ( $\mathrm{Met}^{\mathrm{d}}$ signalling dead receptor [15]), modifying the amino-acids surrounding these tyrosines can alter the selective affinity for some cytoplasmic effectors (Figure 1A) [9]. By converting the two docking sites into optimal binding sites for PI3K ( $\mathrm{Y}_{1349 /}$ $\left.{ }_{1356} \mathrm{MDMS}\right)$ or for $\mathrm{Src}\left(\mathrm{Y}_{1349 / 1356} \mathrm{EEI}\right)$, the $\mathrm{Met}^{2 \mathrm{P}}$ and $\mathrm{Met}^{2 \mathrm{~S}}$ receptors, respectively, were designed to determine the impact of imposing the cytoplasmic effector through which Met can signal, thus raising its contribution above generic signalling levels (Figure 1A). These specificity- switch mutants have been instrumental in addressing the relevance of selective pathways in several biological contexts [9,23-26]. Regarding the function of HGF/Met that triggers myoblast delamination and migration towards limb buds [13-15], the $\mathrm{Met}^{2 \mathrm{P}}$ and $\mathrm{Met}^{2 \mathrm{~S}}$ specificity-switch mutants have limited ability to elicit long-range myoblast migration (Additional file 1: Figure S1A) [9]. In spite of this, the small number of migrating myoblasts is nevertheless sufficient to account for the formation of a substantial amount of muscle in both forelimbs and hindlimbs (Additional file 1: Figure S1A and Figure 2B), and does not affect $\mathrm{MN}$ numbers prior to the onset of muscle dependency for survival (Figure 2: lumbar MNs; Additional file 1: Figure S1B: brachial MNs) [16]. This allelic series of met mutants thus represents an excellent toolbox to uncouple the effects of loss of muscle from direct functions of Met in MNs. Here, we took advantage of the differences in signalling competences of these two versions of Met to determine the degree of flexibility versus specificity for a given biological outcome, such as those elicited by HGF/Met in selective subsets of Met-dependent MN pools (Figure 1B).

\section{Distinct signalling requirement for nerve patterning}

HGF, initially provided by the limb mesenchyme and later by the muscles themselves $[19,27,28]$, is known to promote growth, to exert chemoattractant activity $[17,18]$ and to pattern nerve branching of motor axons $[12,17,18]$. However, a number of abnormalities in peripheral nerve patterns observed in met or hgf loss-of-function mutants $[12,17,18]$, result from secondary effects of the absence of muscles, from the subsequent depletion of muscle-derived guidance signals and from early changes in MN specification [16]. Thus, in spite of extensive documentation of constitutive knockout phenotypes $[9,17,18]$ and our own conditional studies bypassing requirements prior to NOCD [4], formal in vivo demonstration of a direct requirement for Met in axon growth and path-finding is still currently lacking. We have previously shown that neuralspecific Met excision with a Nestin-cre driver was only complete by the end of the NOCD period [4], preventing use from addressing the possibility of a direct requirement for axon growth and guidance prior to NOCD using a conditional approach. Thus, our paradigm using the specificity-switch mutants is suitable for addressing this question.

We previously reported that HGF/Met signalling influenced axon growth in the CM muscle [9]. However, guidance of $C M \mathrm{MN}$ axons to their target does not reflect a direct function of HGF/Met in axon growth, as it is conditioned by an early specification program establishing Pea3 expression domain in a $\mathrm{MN}$ pool, which is under the control of Met as well (see later). To circumvent these caveats and examine signalling modalities of 
A

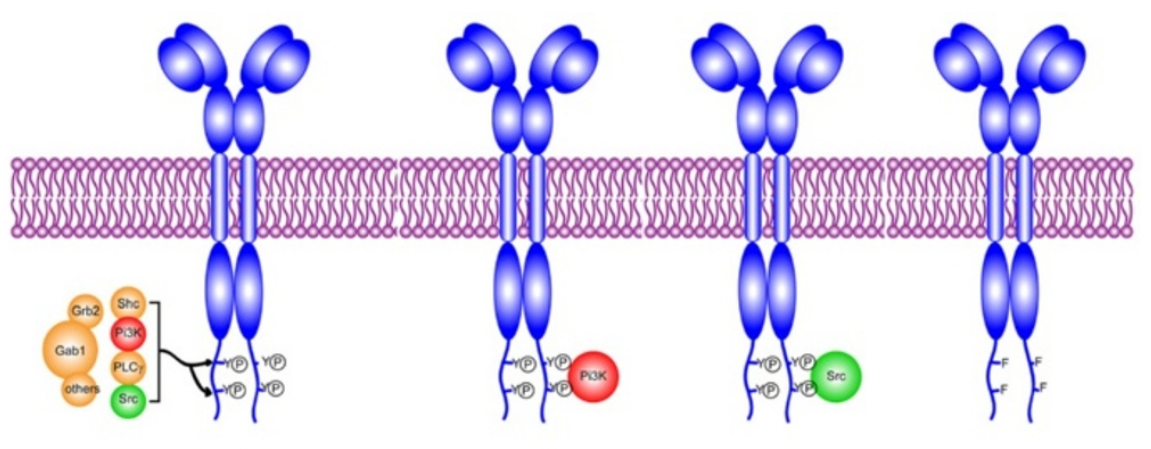

$$
\begin{array}{ccc}
\text { Met }^{\text {WT }} & \text { Met }^{2 \mathrm{P}} & \text { Met }^{2 \mathrm{~S}} \\
\begin{array}{c}
\text { Multifunctional } \\
\text { binding sites }
\end{array} & \begin{array}{c}
\text { 2 optimal PI3K } \\
\text { binding sites }
\end{array} & \begin{array}{l}
\text { 2 optimal } \\
\text { binding si }
\end{array} \\
& \text { Met }^{\text {WT }} & \mathrm{Y}_{1349} \text { VHVNATY }_{1356} \text { VNV } \\
\text { Met }^{2 \mathrm{P}} & \mathrm{Y}_{1349} \text { MDMSATY }_{1356} \text { MDMS }^{2} \\
\text { Met }^{2 \mathrm{~S}} & \mathrm{Y}_{1349} \text { EEINATY }_{1356} \text { EEI } \\
\text { Met }^{\mathrm{d}} & \mathrm{F}_{1349} \text { VHVNATF }_{1356} \text { VNV }
\end{array}
$$$$
\text { Met }^{d}
$$

2 optimal Src

B

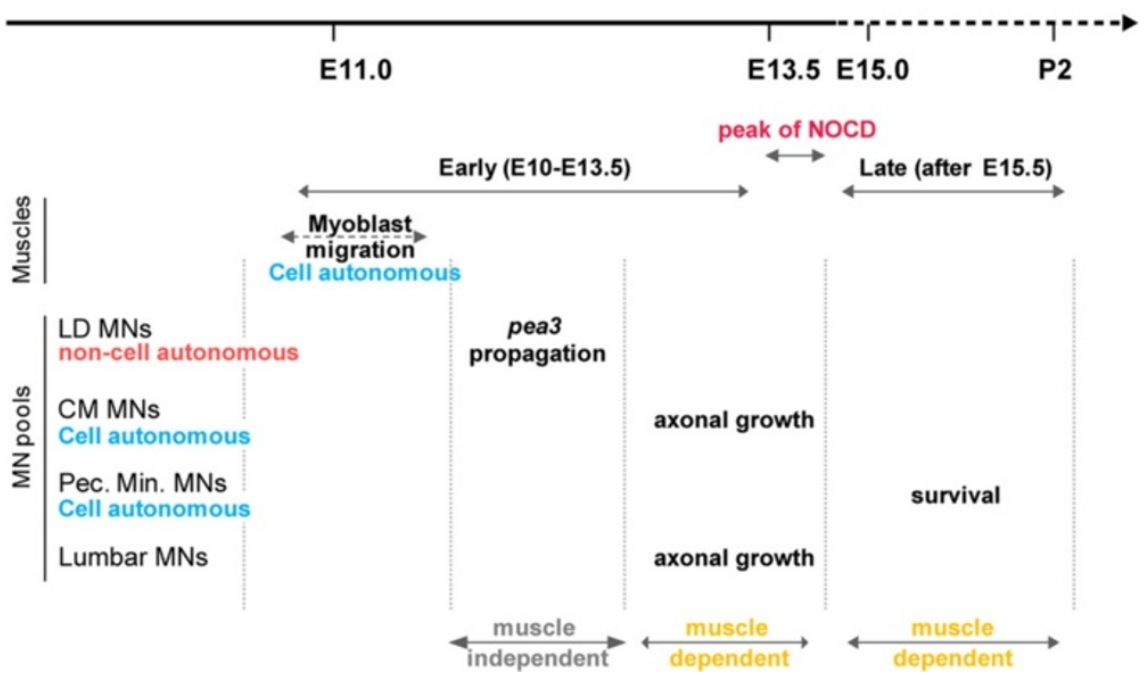

Figure 1 Using Met specificity-switch mutants to study plasticity of HGF/Met functions in motor neurons. (A) The wild type (WT) and three signalling mutant versions of the Met receptors used in this study. The WT receptor (Met ${ }^{W T}$ ) conveys intracellular signalling mainly through two tyrosine residues located in the C-terminal tail. Upon HGF binding and phosphorylation by the Met kinase domain, these tyrosines recruit several cytoplasmic signalling effectors, including Gab1, PI3K, Src, Grb2 and a number of others. The specificity-switch mutants Met ${ }^{2 \mathrm{P}}$ and Met ${ }^{2 \mathrm{~S}}$ have been designed to carry optimal binding sites for PI3K and Src, so as to favour their respective recruitment/activation [9]. The Met ${ }^{d}$ receptor has its two tyrosines replaced by phenylalanines and is thus signalling incompetent $[9,15]$. As a consequence, met ${ }^{d / d}$ embryos have phenotypes indistinguishable from null mutants. (B) Time course of developmental events regulated by HGF/Met signalling that influence MN biology, summarising our earlier findings $[4,16]$. The period shown is divided into an early period, from E10.5 to E13.5, before synaptic contact between MNs and muscles, and a late period, during which MNs are dependent on muscle-derived trophic factors for their survival. The transition between the two periods is marked by a pronounced peak of MN death (NOCD), indicating numerical adjustment of MN numbers to target muscle size. During the muscle-independent period, before NOCD, HGF/Met signalling controls migration of limb myoblasts, axon growth of a subset of Met-expressing MNs, cell autonomous induction of runx1 [4] and non-cell autonomous propagation of Pea3-expression [16]. After NOCD, HGF/ Met controls the survival of a distinct pool of MNs, innervating the pectoralis minor muscle, while it is no longer required for trophic support of CM or latissimus dorsi (LD) MN pools. 


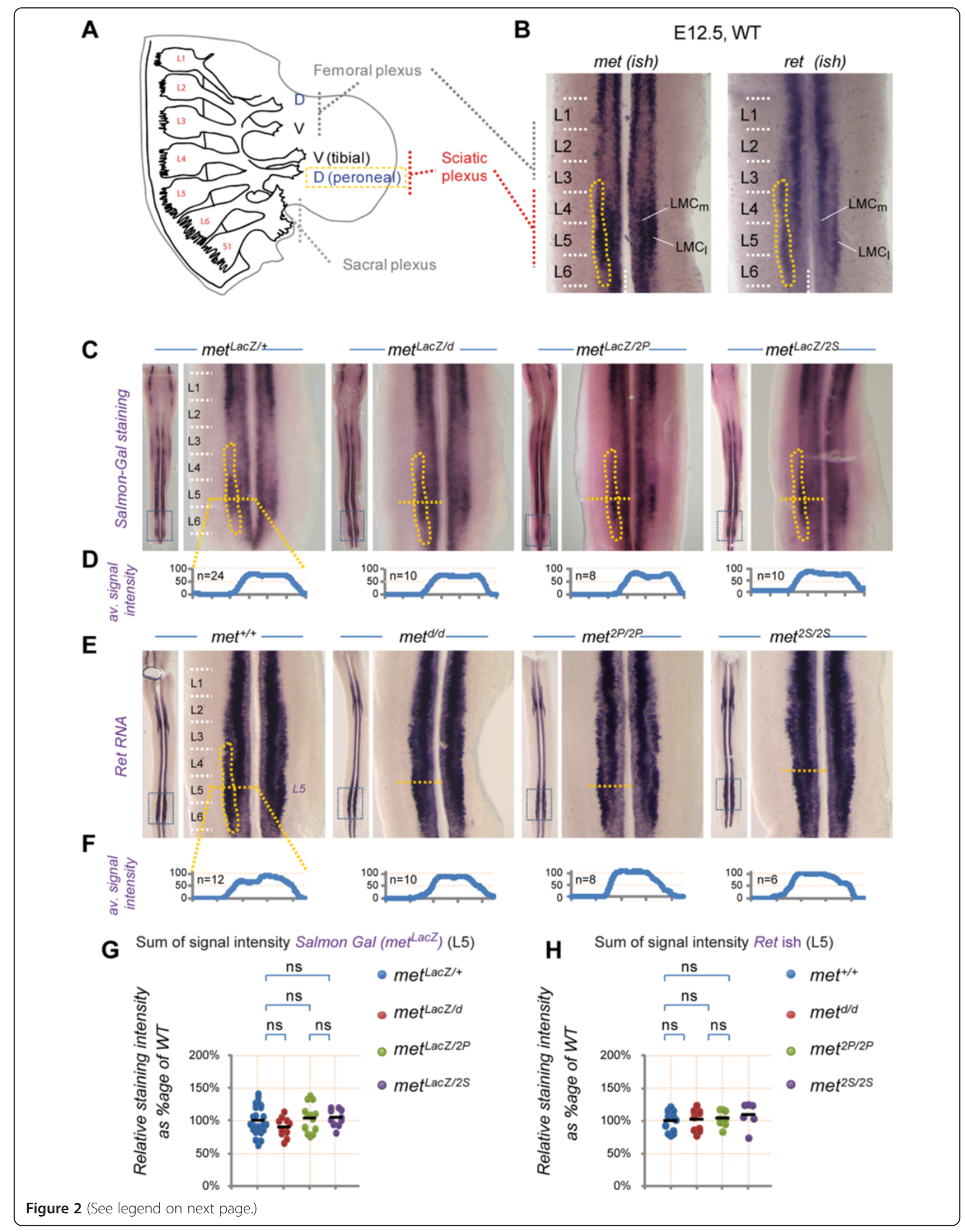


(See figure on previous page.)

Figure 2 Met signalling is required for hindlimb nerve patterning prior to the onset of muscle-dependency for MN survival. (A) Lumbar spinal nerves and plexuses at hindlimb levels based on a lateral view of an E11.5 WT embryo. The sciatic plexus (from which the tibial (ventral) and peroneal (dorsal) nerves emerge) receives axons mostly from L4 and L 5 spinal segments, while the femoral plexus (anterior) collects axons mostly from L2 and L3 spinal segments. (B) ISH with Met and Ret probes on E12.5 WT spinal cords. The LMC pool corresponding to peroneal MNs is indicated in yellow, spanning from mid L3 to L6. (C) Met expression was followed by Salmon-Gal staining in spinal cords from E12.5

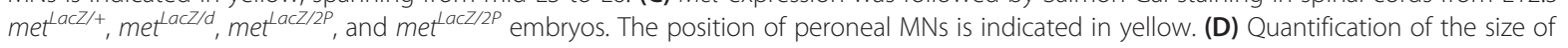
Met-expressing MN pools along the orange line at L5 level. Each plot represents the average signal distribution of the indicated number of spinal cord sides (met ${ }^{L a c Z /+}: n=24 ;$ met $^{L a c Z / d}: n=12 ;$ met $^{\text {LacZ/2P. }}: n=8$; met $^{L a c Z / 2 P}: n=10$. (E) Ret expression was followed by ISH in lumbar spinal cords from E12.5 WT, met ${ }^{d / d}$, met ${ }^{2 P / 2 P}$, met ${ }^{2 S / 2 S}$ embryos. (F) The size of the Ret-expressing MN pools was quantified along the orange line at L5 level. Each plot represents the average signal distribution of the indicated number of spinal cord sides $\left(m e t^{+/+}: n=12 ; m e t^{d / d}: n=10 ; m e t^{2 P / 2 P}: n=8 ; m e t^{2 S / 2 P}\right.$ : $\mathrm{n}=6) .(\mathbf{G}, \mathbf{H})$ Quantifications and statistical analyses of the sum of signal intensity corresponding to measurements of met ${ }^{\text {lacz }}$ expression (G) or Ret ISH staining (H) based on intensity plots in (D) and (F), respectively. The numbers of samples are as indicated in (D) and (F). At E12.5 the size of the MN population is not significantly altered by the reduced muscle mass in met ${ }^{d / d}$, met ${ }^{2 P / 2 P}$, and met ${ }^{2 S / 2 P}$ embryos, indicating that this stage precedes the phase of $M N$ death.

HGF/Met in a system where they exert direct control of axonal growth and guidance, we chose to investigate the motor nerve pattern in the dorsal hindlimb. While Met expression in brachial spinal cord segments is restricted to the Pea3-expressing MN pool, in which HGF/Met controls cell fate specification, Met is also expressed in pools of lumbar MNs, including medial and lateral LMC MN columns at sciatic levels L4 to L6, in which Pea3 is not co-expressed (Figure 2A,B) [19]. We first established that at E12.5, prior to the onset of cell death caused by trophic deprivation, no difference in the amount of Retexpressing MNs in the lumbar segments could be detected in any of the met mutant genotypes ( $m e t^{d / d}$, $m e t^{2 P / 2 P}$ and $m e t^{2 S / 2 S}$ ) compared to controls (Figure 2E,F,H). Further confirmation came from analysis of met-expressing neurons directly, by following one copy of the $m e t^{\text {Lac } Z}$ reporter/knockout allele, in $m e t^{L a c Z /+}, m e t^{L a c Z / d}, m e t^{L a c Z / 2 P}$ and $m e t^{L a c Z / 2 S}$ embryos (Figure 2C,D,G).

We next performed careful correlative analysis of nerve and muscle patterns in the dorsal hindlimb, using whole-mount anti-neurofilament staining and MyoD in situ hybridisation (ISH). In WT embryos, the dorsal shank is mainly innervated by the peroneal nerve branch emerging from the sciatic plexus (Figure 2A) [29]. This nerve follows a stereotyped trajectory, first crossing the extensor digitorum longus (EDL) muscle on its proximal extremity, and continuing its distal progression towards the foot in the space between the EDL muscle and the tibialis anterior (TA) muscle (see scheme in Figure 3D). In wild-type (WT) embryos, the sharp turn made by the peroneal nerve between the portion crossing the EDL muscle and the longitudinal portion running between $E D L$ and $T A$ muscles consistently defines an angle $(\alpha)$ of approximately $120^{\circ}$ on average. Furthermore, at the contact site between the nerve and each muscle, a few axonal side branches leave the nerve to enter the muscle where they will later establish synapses with muscle fibres (Figure 3D, blue arrows, see also Additional file 2: Figure S2E). In $m e t^{d / d}$ embryos, in the absence of limb muscles, the peroneal nerve displayed no detectable side branches and a significantly altered shape (Figure 3A,C), running straight through the limb with $\alpha$ increased to approximately $160^{\circ}$ on average (Figure $3 \mathrm{E}$, $* * 0.001$ ). Although we found reduced amounts of Pea3-expressing $\mathrm{MNs}$ in the lumbar region (Additional file 2: Figure S2C), this peroneal nerve phenotype is not a consequence of altered Pea3 expression, as: (a) Pea3 is not co-expressed with $m e t$ in peroneal MN pools at L4-L5 levels (yellow dotted pools, Additional file 2: Figure S2B), and (b) Pea3-null mutants exhibit an intact peroneal nerve shape (Additional file 2: Figure S2D). Thus, the peroneal nerve shape is an appropriate model for unambiguously establishing Met-signalling requirements for axonal growth, without possible consequences related to defects in muscle formation and/or MN specification.

We first established a cartography of developing muscles in $m e t^{2 P / 2 P}$ and $m e t^{2 S / 2 S}$ dorsal hindlimbs and found an asymmetric pattern, with the posterior muscles (including peroneus longus and peroneus digiti) showing a reduced mass but almost normal shape, while muscles of the anterior group (including $E D L$ and $T A$ muscles) were severely reduced in size, and the distal muscle mass was completely absent (Figure 3B,C,G). The anteriormost muscle, $T A$, was the most affected, and its range of phenotypical variations included a complete absence in a small subset of embryos. The overall quantification of its size revealed no significant difference between $m e t^{2 P / 2 P}$ and $m e t^{2 S / 2 S}$ embryos. There was also no significant difference in the size of the EDL until E14.5, when secondary myogenesis starts [30]. While examining the nerve pattern, we found that the peroneal nerve of $m e t^{2 P / 2 P} \mathrm{em}$ bryos had a shape similar to that of control embryos, with an average $\alpha$ of approximately $135^{\circ}$, and its side branches in contact with $E D L$ and $T A$ were present in most cases (with an average number of eight side branches per nerve compared to nine per nerve in controls; Figure 3F). In contrast, the peroneal nerve shape of $m e t^{2 S / 2 S}$ embryos was identical to that seen in $m e t^{d / d}$ mutants, with an 


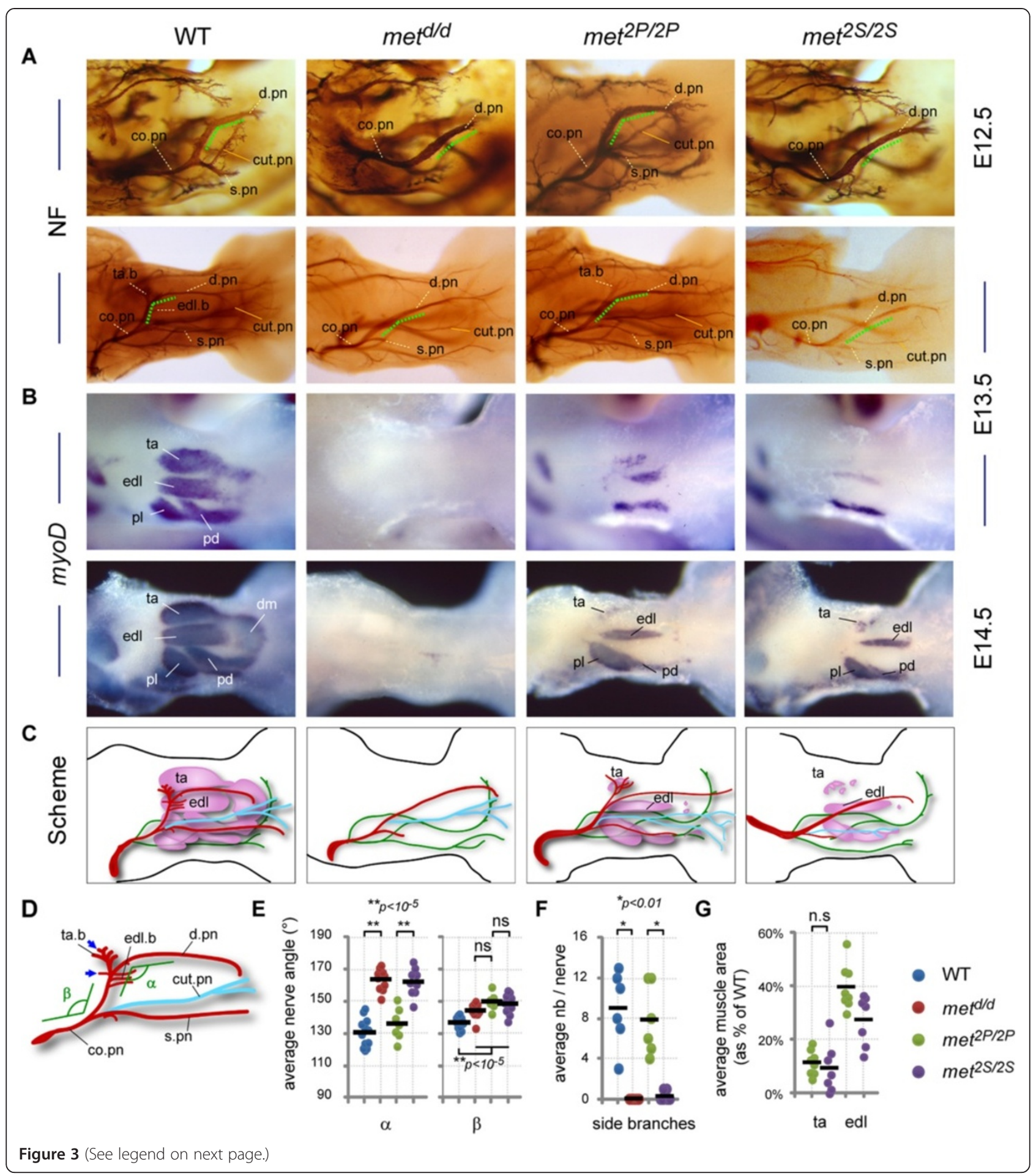


(See figure on previous page.)

Figure 3 Uncoupling muscle-dependent from Met-dependent axonal branching. (A) Anti-neurofilament (NF) immunohistochemistry on E12.5 and E13.5 hindlimbs of WT and met mutants. (B) MyoD ISH showing differentiating muscles in E13.5 and E14.5 hindlimbs. (C) Recapitulative schemes positioning hindlimb muscles and nerves with respect to each other. Colour code for nerves; green: ventral nerves not in the focal plane and unaffected by lack of Met signalling; red: peroneal nerve motor branches, innervating the dorsal limb compartment, corresponding to the common peroneal nerve as it emerges from the sciatic plexus. The cutaneous sensory peroneal nerve branch is shown in blue. (D) Quantification parameters. a: angle between the transverse and distal portion of the deep peroneal nerve. $\beta$ : angle between common and deep peroneal nerve portions, used as internal morphological landmark. Blue arrows: contact sites with ta and edl muscles where the number of peroneal nerve side branches were quantified. (E) The nerve phenotype severity was assessment by measuring the $a$ and $\beta$ angles, in WT ( $\mathrm{n}=12$ ), $m e t^{d / d}$ ( $\mathrm{n}=10$ ), $m_{e t^{2 P / 2 P}}(n=7)$, and $m^{2 S / 2 S}(n=9)$ E12.5 and E13.5 hindlimbs. Each dot represents one embryo side. The same colours representing the genotypes were used in all graphs. (F) Plot showing the respective numbers of side-branches per peroneal nerve (pooling ta and edl branches), in E13.5 WT $(n=6)$, met $^{d / d}(n=7)$, met $^{2 P / 2 P}(n=6)$, and met $^{2 S / 2 S}(n=8)$ hindlimbs. Differences between WT and met ${ }^{d / d}$ embryos, and between met ${ }^{2 P / 2 P}$ and met $^{2 S / 2 S}$ embryos, respectively, are significant (Mann-Whitney test, $p<0.01)$.) (G) Measurements of ta and edl muscle surface were performed on WT ( $\mathrm{n}=6$ ), $m^{d / d}(n=6), m^{2 P / 2 P}(n=8)$, and $m^{2 S / 2 S}(n=7)$ E13.5 and E14.5 hindlimbs stained with MyoD ISH. Areas were expressed as percentage of the mean area in WT embyos (represented as 100\%). The plot therefore shows only met ${ }^{2 P / 2 P}$ and met ${ }^{2 S / 2 S}$ values, each dot representing one embryo side.

average $\alpha$ of approximately $160^{\circ}$ and side branches towards $E D L$ and $T A$ never detected. Of notice, another internal morphological landmark, $\beta$, reflecting the turn made by the peroneal nerve emerging from the sciatic nerve, did not exhibit significant differences between $m e t^{d / d}$, $m e t^{2 S / 2 S}$ and $m e t^{2 P / 2 P}$ embryos, although significantly affected by the morphological changes in muscle shape compared to controls. Altogether, these data demonstrate that signalling by $\mathrm{Met}^{2 \mathrm{P}}$, but not by $\mathrm{Met}^{2 \mathrm{~S}}$, can efficiently promote side-branch formation, and shape the peroneal nerve, provided that the corresponding muscle is present in the limb (likely to supply either HGF itself or a cofactor necessary for the growth response). Furthermore, they indicate that distinct signalling cascades, such as those triggered by these two receptor variants, are not equivalent in their capacity to execute nerve patterning by Met.

\section{Signalling cascades effective at mediating Met-triggered Pea3-domain expansion are differentially sensitive to Pea3 gene dosage}

We next investigated the signalling requirement for cell fate specification by focusing on the program that defines which $\mathrm{MN}$ pools express the transcription factor Pea3, in which HGF/Met signalling is required [16]. We have previously shown that the Pea3-expressing $\mathrm{MN}$ population at brachial levels is constituted of two main subgroups of MNs [16]. In the first group of pioneer MNs, Pea3 expression is initially induced by glial-derived neurotrophic factor (GDNF), produced by the target muscle [31]. HGF then acts on the same pioneer MNs, via Met, instructing them to induce Pea3 expression in additional neurons, a feed-forward induction process that leads to lateral and anterior expansion of the Pea3 expression domain (summarised in Additional file 3: Figure S3, see also $[16,31])$. Such biphasic establishment of the Pea3 expression domain is illustrated by the phenotype of $m e t^{d / d}$ mutants, in which this second process is abolished, leaving only the GDNF-dependent population [16].
Importantly, the size reduction of Pea3-expressing motor pools observed in $m e t^{d / d}$ mutants occurred before the onset of muscle dependency and was not caused by muscle depletion, since such a defect was not observed in Pax3deficient $\left(\mathrm{Pax}^{\mathrm{Sp} / \mathrm{Sp}}\right)$ embryos, which also lack limb muscles [16]. Whole-mount ISH with Pea3 on E12.5 spinal cords revealed the Pea3 expression domain in $m e t^{2 P / 2 P}$ and $m e t^{2 S / 2 S}$ spinal cords had a normal shape, contrasting with the reduced shape displayed in $m e t^{d / d}$ mutants (Figure 4A,B,E). We ruled out that the competence of $\mathrm{Met}^{2 \mathrm{P}}$ and $\mathrm{Met}^{2 \mathrm{~S}}$ signalling mutants to mediate expansion of Pea3 expression domain could be indirectly influenced by the reduced muscle content, by showing that: (a) no detectable changes in the amount of Ret-expressing MNs were found in E12.5 met $t^{d / d}$, $m e t^{2 P / 2 P}$ and $m e t^{2 S / 2 S}$ brachial spinal cords (Additional file 1: Figure S1B); (b) the Pea3 domain expansion is independent of muscle-derived signals, and occurs at a stage when the muscle phenotype of $m e t^{d / d}, m e t^{2 P / 2 P}$ and $m e t^{2 S / 2 S}$ embryos is almost equivalent (E11.5) (Additional file 1: Figure S1A; see also [16]); (c) comparably sized Pea3 and Ret expression domains (Additional file 4: Figure S4B,C and Figure 4B) were also observed in the brachial spinal cord of $m e t^{2 P n e o / 2 P n e o}$ and $m e t^{2 S n e o / 2 S n e o}$ signalling-switch mutant embryos still carrying the neo cassette (which had served to engineer the modified alleles, the latter causing attenuated expression levels of the mutant receptors [9] and resulting in further reduced muscle masses (Additional file 4: Figure S4A)). Thus, the signalling routes used by the $\mathrm{Met}^{2 \mathrm{P}}$ and $\mathrm{Met}^{2 \mathrm{~S}}$ specificity-switch mutants are equally sufficient, even when sensitised by lowered receptor expression levels, to execute the mechanisms leading to Pea3 propagation.

The signalling cascades triggered by the $\mathrm{Met}^{2 \mathrm{P}}$ and $\mathrm{Met}^{2 \mathrm{~S}}$ specificity-switch mutants involve distinct, nonequivalent mechanisms [9]. However, besides the selective activation of PI3K and Src pathways, the two receptors also share a common set of signalling effectors, such as the adaptor molecule Gab1 and its downstream 


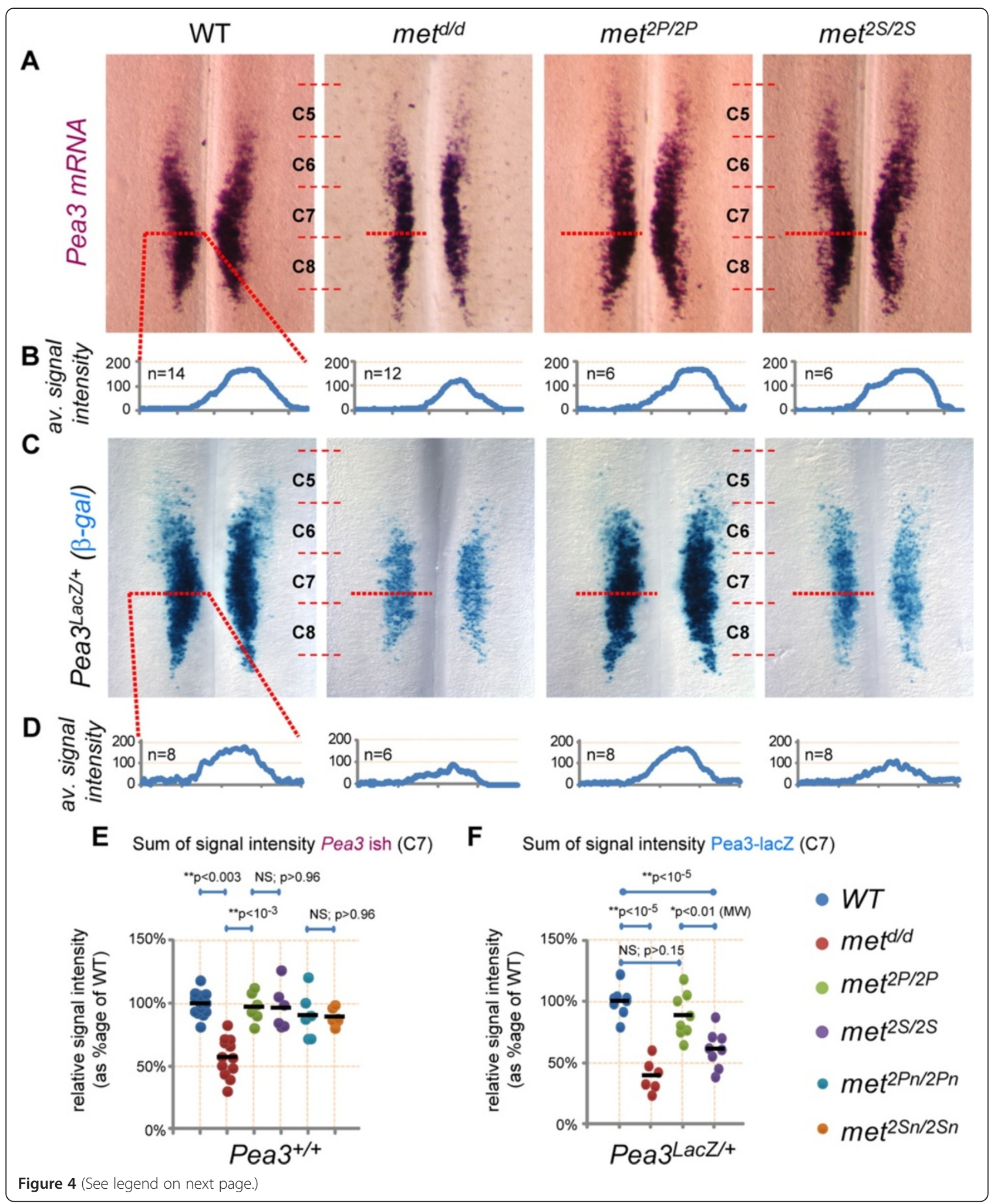


(See figure on previous page.)

Figure 4 Met-induced Pea3 propagation can be executed through PI3K and Src pathways. (A) ISH analysis of the establishment of Pea3 expression domain in spinal MNs. Images show brachial region of flat-mounted E12.5 spinal cords hybridised with Pea3 probes in WT, met ${ }^{d / d}$, met $^{2 P / 2 P}$, and met $^{2 S / 2 S}$ embryos. Whereas Pea3 MN pool is reduced in met ${ }^{d / d}$, it appears normal in met ${ }^{2 P / 2 P}$ and met ${ }^{2 S / 2 S}$ embryos. (B) Quantifications of the lateral expansion of the Pea3 domain by signal intensity analysis: each plot represents the average signal distribution measured on the indicated number of spinal cord sides along the red dotted line as positioned in each image in (A) (WT: $n=14 ; m e t^{d / d}: n=12 ; m e t^{2 P / 2 P}: n=6 ; m e t^{2 S / 2 S}$ : $\left.n=6\right)$. (C): Brachial regions of flat mounted spinal cords stained with X-Gal to reveal $\beta$-galactosidase activity of the Pea3 ${ }^{\text {LacZ }}$ knock-in. This context with only one functional copy of Pea3 is sufficient for complete Pea3 propagation by Met $^{2 P}$, but is not permissive for Met ${ }^{2 S}$ receptor. There was a lack of propagation in met ${ }^{d / d}$ : Pea $3^{L a c z /+}$ as compared to met $^{+/+}$:Pea $3^{\text {LacZ/+ }}$ spinal cords. (D) Quantifications of the lateral expansion of the Pea3/ $\beta$-galactosidase-positive domain by signal intensity analysis: each plot represents the average signal distribution as measured on the indicated number of spinal cord sides (WT: $n=8 ;$ met $^{d / d}: n=6 ;$ met $^{2 P / 2 P}: n=8 ;$ met $^{2 S / 2 S}$ : $n=8$ ) along the red dotted line as positioned in each image in $(\mathbf{C})$. (E, $\mathbf{F})$ Quantifications and statistical analyses of the sum of the signal intensities corresponding to measurements of

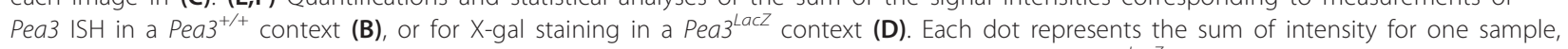
the number of samples being as indicated in (B) and (D). The absence of one Pea3 functional copy in a Pea3 ${ }^{\text {LacZ }}$ context reveals a significant difference in Pea3 dosage requirement between met $^{2 P / 2 P}$ and met $^{2 S / 2 S}$ embryos.

signalling components [9], which could account for their equivalent capacity to induce Pea3 propagation. To discriminate between activation of selective pathways versus background levels of shared downstream effectors, we asked whether the signalling mechanisms in $m e t^{2 P / 2 P}$ and $m e t^{2 S / 2 S}$ embryos could be genetically distinguished. We reasoned that a way to address this issue was provided by the fact that the process of expansion of Pea3 expression involves a positive reinforcement loop during which met and Pea 3 are necessary for each other's expression (see Additional file 3: Figure S3) [16]. Indeed, we have shown that Pea3 itself contributes to its own propagation, since it is necessary to induce Met expression within the pioneer domain, as shown in $\mathrm{Pea}^{-/-}$mutants [16]. Consistently in met loss-of-function mutants, the reduced expansion of the Pea3 domain also results in reduced met expression, as assessed by comparing $\beta$-galactosidase activity in $m e t^{L a c Z /+}$ and $m e t^{L a c Z / d}$ embryos (Additional file 1: Figure $\mathrm{S} 1 \mathrm{D}, \mathrm{G})$, whereas expansion of $m e t^{L a c Z}$ expression was fully executed in $m e t^{L a c Z / 2 P}$ and $m e t^{L a c Z / 2 S}$ embryos, paralleling the intact expansion of Pea3 expression domain (Additional file 1: Figure S1D,G). We therefore challenged the robustness of the MN pool specification function mediated by the Met signalling-switch mutants, by genetically lowering Pea3 expression levels. We took advantage of the $P e a 3^{\text {LacZ }}$ knockout allele, in which the insertion of the LacZ gene not only results in loss of Pea3 functions, but also recapitulates the Pea3 expression domain [32]. Intriguingly, we found that whereas $m e t^{2 P / 2 P} ; \mathrm{Pea}^{\mathrm{LacZ} /+}$ had a pattern of $\beta$-galactosidase activity similar to Pea $3^{\mathrm{LacZ/+}}$ control E12.5 brachial spinal cords, $\mathrm{met}^{2 \mathrm{~S} / 2 \mathrm{~S}}$; Pea $3^{\mathrm{LacZ} /+}$ displayed a significantly reduced number of blue MNs, resulting in a phenotype similar to that of $m e t^{d / d}$; $P e a 3^{\text {LacZ/+ }}$ mutants (Figure 4C,D,F). Thus although switching signalling cascades activated by Met mutants leads to comparable efficacy to expand the Pea3 expression domain in the context of two functional copies of Pea3, the signalling routes employed by the Met signalling variants are differentially sensitive to Pea3 gene dosage reduction: the $\mathrm{Met}^{2 \mathrm{~S}}$ route is sensitive, whereas the $\mathrm{Met}^{2 \mathrm{P}}$ is not. This indicates that higher Pea3 levels are required for its own propagation of expression when mediated through a $\mathrm{Met}^{2 \mathrm{~S}}$ /Src-dependent mechanism, whereas lower Pea3 doses are sufficient when propagation occurs via a $\mathrm{Met}^{2 \mathrm{P}}$ / PI3K-dependent cascade. Thus, the program for acquisition of Pea3 identity triggered by HGF/Met allows a certain degree of signalling flexibility, and the competence of alternative pathways to execute this function is differentially dependent on the genetic dosage of Pea3.

\section{Plasticity of Met signalling requirement for a cell autonomous survival response in primary motor neuron cultures}

We next explored the plasticity of the MN survival supporting function of HGF/Met when challenged with signalling switches. HGF is known to promote the survival of subsets of limb-level MNs in culture $[4,18,19]$. In vivo, the HGF/Met requirement for MN survival is limited to lumbar MNs in chicks [33] and to a selective pool of brachial MNs innervating the pectoralis minor muscle in mice, as shown with neural-specific ablation of Met [4], prompting us to focus our studies on this $\mathrm{MN}$ pool. Studying the dependency on HGF/Met for MN survival requires distinguishing its direct trophic function from the indirect consequences of the trophic factors for depletion resulting from myoblast migration defects. Bypassing the consequences of decreased muscle mass in the limbs of met-signalling mutant embryos can be achieved in vitro using primary MN cultures and in vivo through neuronal lineage-restricted signalling modulation of Met properties.

As a first approach, we assayed survival in primary MN cultures established as described [34] from WT or met-signalling mutant embryos at E12.5, a stage when $\mathrm{MN}$ numbers were not affected yet by reduced muscle mass (see Figure 3 and Additional file 1: Figure S1B). E12.5 primary MNs were collected and pooled from brachial and lumbar spinal cords, and then cultured in the presence of either GDNF (100 pg/ml) or HGF $(0.4 \mathrm{ng} / \mathrm{ml}$ 
or $2 \mathrm{ng} / \mathrm{ml}$ ) for 3 days. As previously reported for rat MNs, both factors prevented the death of a proportion of WT limb innervating MNs (Figure 5A,B: WT) [4,19,35]. Since MNs derived from $m e t^{d / d}, \mathrm{met}^{2 P / 2 P}$, and $m e t^{2 S / 2 S}$ embryos retained their ability to respond to GDNF (Figure 5A) and to BDNF (data not shown), the response to HGF was expressed as a percentage of GDNF-induced survival, offering the possibility to normalise all experiments performed. While $m e t^{d / d} \mathrm{MNs}$ no longer responded to HGF, MNs derived from $m e t^{2 P / 2 P}$ and $m e t^{2 S / 2 S}$ mutants exhibited a survival response to HGF similar to that of WT embryos (Figure 5B). As reported for the rat, WT thoracic and sacral MNs required a higher concentration of HGF $(10 \mathrm{ng} / \mathrm{ml})$ to survive [19]. We found that MNs purified from thoracic and sacral regions (non-limb-innervating MNs) from $m e t^{2 P / 2 P}$ and $m e t^{2 S / 2 S}$ but not from $m e t^{d / d}$ embryos survived in response to HGF treatment (data not shown). Since at the stage at which MNs are collected for these primary cultures (E12.5) most Metexpressing MNs are equally present in all genotypes, (Figure 3 and Additional file 1: Figure S1), the absence of a survival response by $m e t^{d / d}$ mutant MNs is not a consequence of a complete depletion of met-positive neurons, but indicates that the $\mathrm{Met}^{\mathrm{d}}$ receptor does not transduce survival signals. In contrast, cultures from $m e t^{2 P / 2 P}$ and $m e t^{2 S / 2 S}$ spinal cords started with an unaltered Met-responsive population compared to WTs (Figure 3, Additional file 1: Figure S1, Additional file 5: Figure S5). As their survival in response to HGF was comparable to survival of WT MNs, this indicates that the $\mathrm{Met}^{2 \mathrm{P}}$ and $\mathrm{Met}^{2 \mathrm{~S}}$ receptors mediate the survival induced by HGF as efficiently as the WT receptor. Altogether, these results establish that signalling routes solicited by $\mathrm{Met}^{\mathrm{WT}}, \mathrm{Met}^{2 \mathrm{P}}$ or $\mathrm{Met}^{2 \mathrm{~S}}$ are equally capable of triggering in vitro $\mathrm{MN}$ survival by HGF.

The ability of the $\mathrm{Met}^{2 \mathrm{P}}$ receptor to mediate a survival response to HGF can be understood as the recruitment and activation of the PI3K-Akt pathway, which is well known to be involved in the inhibition of apoptosis [36]. In contrast, the survival function triggered by $\mathrm{Met}^{2 \mathrm{~S}}$ suggests a novel link between Src and a survival pathway in MNs. We therefore asked whether activation of Src mediated the survival response either by converging onto the PI3K-Akt pathway (as reported for GDNF-induced neuronal survival [10]), or by acting through an independent mechanism. This question was addressed by pharmacologically inhibiting the PI3K pathway or the Src family kinases with the selective inhibitors LY294002 or PP2, respectively, at concentrations that do not present toxic effects on the basal survival (LY294002: $1 \mu \mathrm{M}$; PP2: $0.2 \mu \mathrm{M}$; data not shown). We found that the survival responses mediated by $\mathrm{Met}^{2 \mathrm{P}}$ or by $\mathrm{Met}^{2 \mathrm{~S}}$ were selectively abolished by LY294002 and by PP2, respectively (Figure 5D), but not by PP3, a non-functional analogue of
PP2 (data not shown). These findings argue that the MN survival response triggered by $\mathrm{Met}^{2 \mathrm{P}}$ or $\mathrm{Met}^{2 \mathrm{~S}}$ specificityswitch mutants are indeed mediated by PI3K or Src, respectively, as they were designed to do [9]. Using PP2 and LY294002, we further showed that signalling by Met ${ }^{2 \mathrm{P}}$ does not require Src activity for survival, whereas the survival response elicited by $\mathrm{Met}^{2 \mathrm{~S}}$ does not require PI3K activity (Figure 5D). Interestingly, pharmacological inhibition of Mek by PD98059 prevented the survival response elicited by $\mathrm{Met}^{2 \mathrm{~S}}$, but not by $\mathrm{Met}^{2 \mathrm{P}}$ (Figure 5D). Together, these experiments indicate that the MN survival promoted by these specificity-switch receptors occurs through activation of distinct sets of signalling pathways. The survival response triggered by $\mathrm{Met}^{2 \mathrm{P}}$ in $\mathrm{MNs}$ is mediated through an efficient activation of PI3K, and required neither SrC family kinases nor the Mek pathway. In contrast, the mechanism by which $\mathrm{Met}^{2 \mathrm{~S}}$ mediates the survival response does not involve the traditional PI3K-Akt survival pathway, but rather activation of both Src family kinases and the Mek pathway. Intriguingly, we found that all three inhibitors abolished the survival response to HGF by WT Met in MNs (Figure 5D). This indicates that unlike the mutant receptors, for which altered docking sites have forced the choice of one respective signalling pathway, in a WT context, Met uses a combination of these three signalling pathways. Altogether, the combination of our genetic and pharmacological approaches indicates that in vitro there is a remarkable plasticity in the signalling requirements for MNs survival, which can be achieved equally efficiently by alternative pathways permissive for promoting survival (Figure 5E).

\section{Plasticity of signalling effectors for Met-dependent motor neuron survival in vivo}

We next sought to determine whether such flexibility in signalling requirements for HGF/Met-dependent $\mathrm{MN}$ survival also occurs in vivo. In contrast to early stages, once MNs have become dependent on muscle-derived signals for survival, depletion of limb muscles in met-signalling mutants causes limb MN death [16]. This is illustrated by the disappearance of Pea3-expressing neurons (Figure 6A) and the drastic reduction in the number of Ret-expressing MNs occurring in met $^{d / d}$ embryos at E14.5 (Figure 6B), as well as by the proportional reductions of Pea3-expressing or Ret-expressing MNs in the brachial spinal cord of $m e t^{2 P / 2 P}$ and $m e t^{2 S / 2 S}$, $m e t^{2 P n e o / 2 P n e o}$ and $m e t^{2 S n e o / 2 S n e o}$ embryos with reduced volumes of muscles (Figure 6, Additional file 1: Figure S1A, and Additional file 4: Figure S4A,D,E). Therefore, the process of $\mathrm{MN}$ death that matches the number of MNs with the number of myofibres in the cognate target muscle, occurs between E12.5 and E14.5. This constitutes genetic dating of the onset of muscle dependency, consistent with previous observations $[4,16]$. 

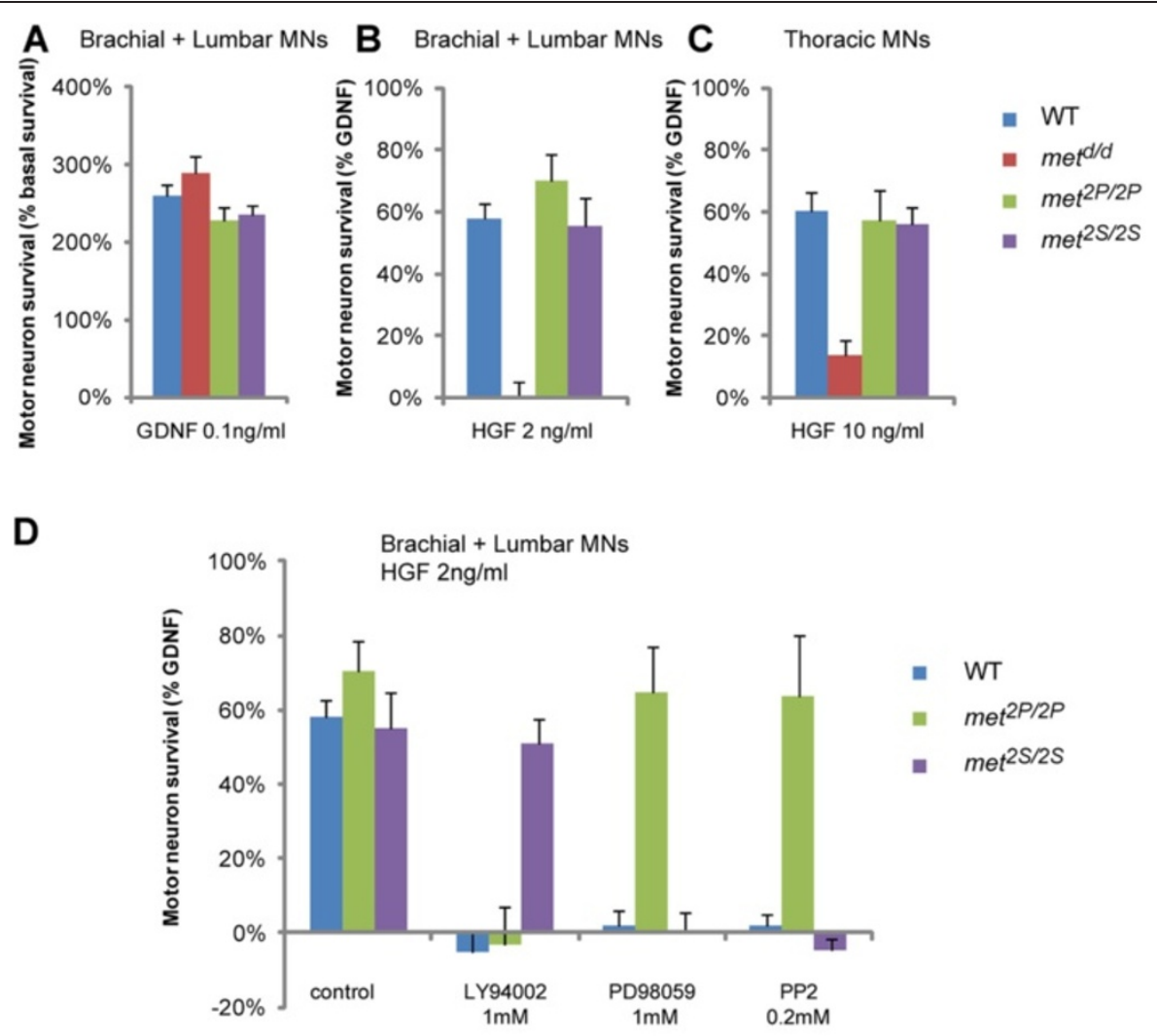

\section{E signalling for HGF-induced MN survival}
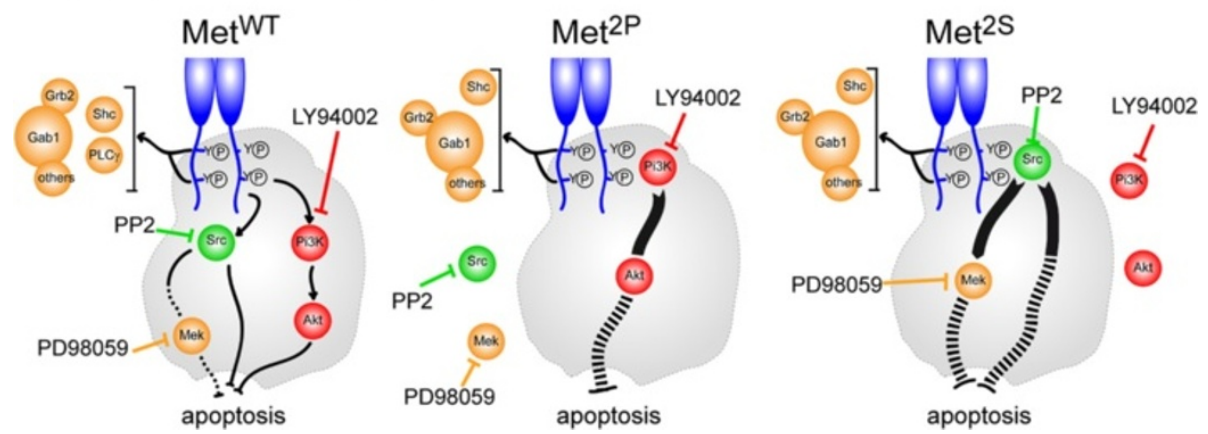

Figure 5 Cell autonomous regulation of MN survival by Met can be achieved through distinct signalling pathways. (A) In vitro

assessment of survival of MNs derived from WT, met ${ }^{2 P / 2 P}$, met ${ }^{2 S / 2 S}$, and met ${ }^{d / d} \mathrm{E} 12.5$ embryos in the presence of GDNF (100 pg/ml). Survival values are expressed as percentage of the survival in basal medium (defined as 100\%). The ability of MNs to respond to GDNF is not altered in met ${ }^{d / d}$, $m e t^{2 P / 2 P}$, and $m e^{2 s / 2 S}$ mutants. Therefore, in subsequent panels, values are normalised with the survival in basal medium (defined as $0 \%$ ) and expressed as percentage of their response to GDNF (defined as 100\%). (B,C) Survival of brachial + lumbar (B) or thoracic (C) MNs derived from WT, met $t^{2 P / 2 P}, m^{2^{2 S / 2 S}}$, and met ${ }^{d / d}$ E12.5 embryos in the presence of HGF $(2 \mathrm{ng} / \mathrm{ml})$. Whereas met ${ }^{d / d} \mathrm{MNs}$ do not show any survival response to HGF, met $t^{2 P / 2 P}$ and $m e t^{2 S / 2 S} \mathrm{MNs}$ respond as efficiently as WT MNs. (D) Signalling requirements downstream of Met ${ }^{W T}$, Met ${ }^{2 P}$, and Met ${ }^{2 S}$ for MN survival by HGF were explored using inhibitors of PI3K (LY294002: $1 \mu \mathrm{M})$, Mek (PD98059: $1 \mu \mathrm{M})$, and Src (PP2: $0.2 \mu \mathrm{M})$, respectively). Inhibitors were used at concentrations not toxic on the basal survival. All three inhibitors efficiently blocked the survival response of WT MNs to HGF. As expected, the survival response in met $^{2 P / 2 P} \mathrm{MNs}$ was selectively blocked by the PI3K inhibitor. In contrast, the survival response in met ${ }^{2 S / 2 S} \mathrm{MNs}$ was abolished by inhibiting Src or Mek. For each genotype, two to four independent experiments were done in triplicate comparing MNs from mutant embryos and from their WT littermates. (WT: $n=9 ;$, eet $^{2 P / 2 P}: n=3 ;$ met $^{2 S / 2 S}: n=4 ;$ met $\left.^{d / d}: n=2\right)$. Error bars indicate standard error of the mean. (E) Schematic representation of the signalling components involved in blocking apoptosis downstream of the wild-type Met ${ }^{\mathrm{WT}}$ or the specificity switch mutants $\mathrm{Met}^{2 \mathrm{P}}$ and $\mathrm{Met}^{2 \mathrm{~S}}$ receptors. The inhibitors used are indicated. 


\section{E14.5: survival with reduced muscle mass}

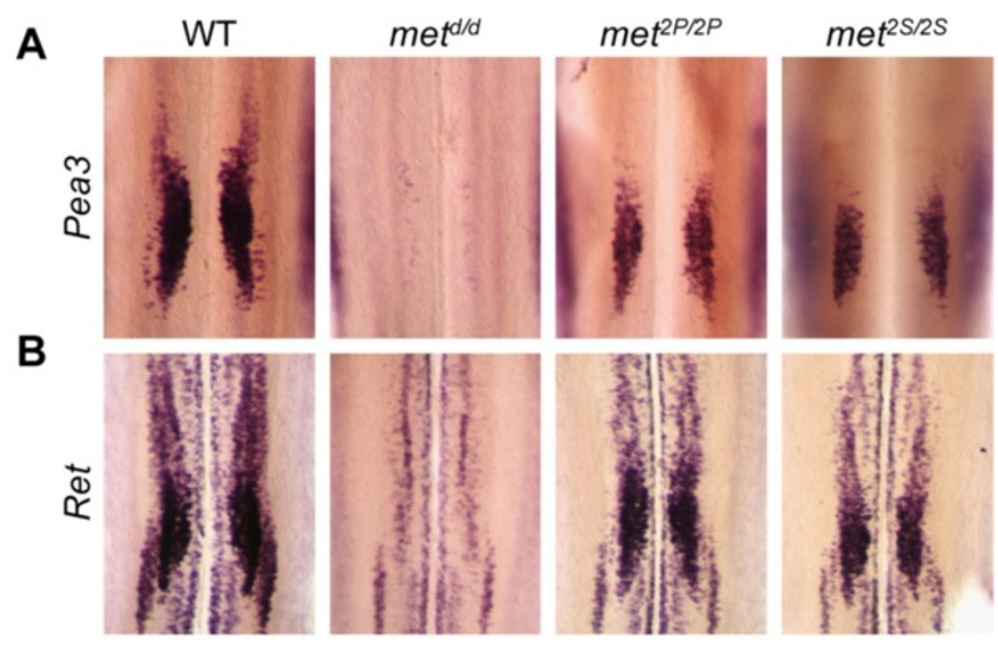

P2: survival with unaltered muscle mass (neuronal-specificity-switch)
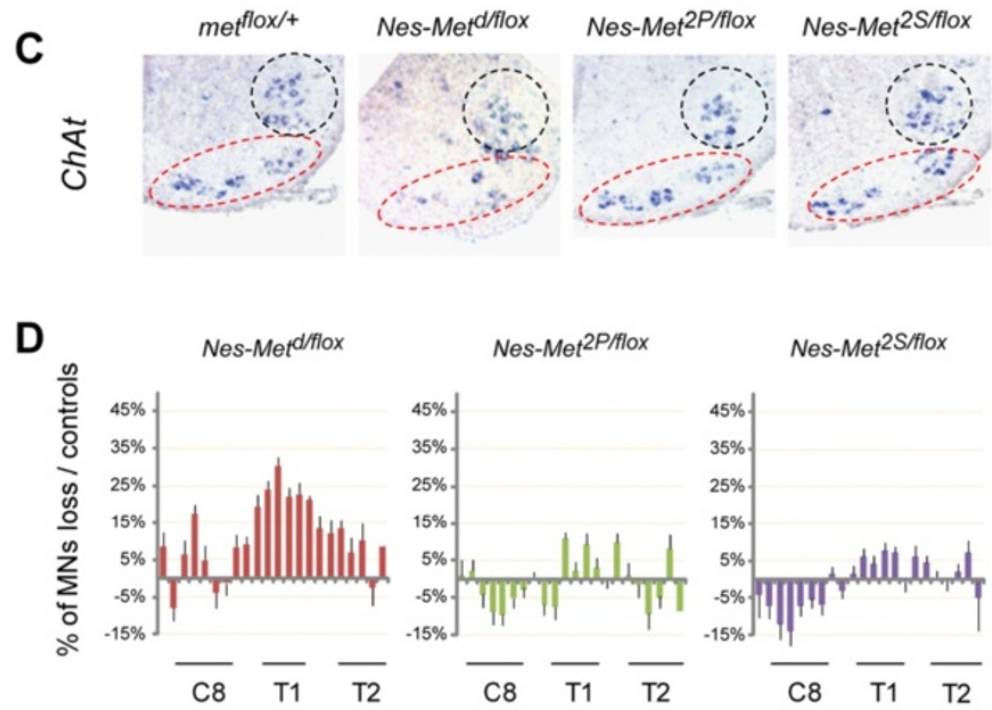

Mann-Whitney indic. comp.

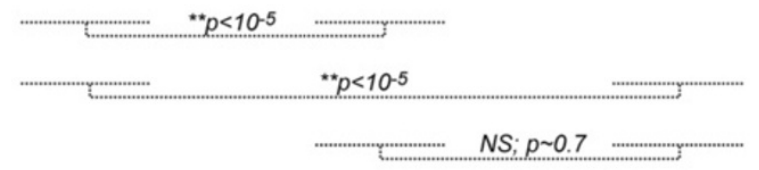

$\begin{aligned} & \text { Mann-Whitney } \\ & \text { (comp. to controls) }\end{aligned} \quad *{ }^{* *} p<10^{-4} \quad$ NS; $p \sim 0,63 \quad$ NS; $p \sim 0,9$

Figure 6 (See legend on next page.) 
(See figure on previous page.)

Figure 6 Conditional neuronal-specificity-switch of Met signalling to PI3K and Src is sufficient for pectoralis minor motor pool survival in vivo. $(\mathbf{A}, \mathbf{B})$ Correlation between $M N$ numbers in spinal cords with the amount of limb muscle in Met signalling mutants (see Figure $2 \mathrm{~A}, \mathrm{~B}$ ). Analysis of MN pool survival in vivo after the onset of muscle dependency by Pea3 (A) or Ret (B) ISH on spinal cords from E15.5 WT, met ${ }^{d / d}$, $m e^{2 P / 2 P}$, and $\mathrm{met}^{2 S / 2 S}$ embryos ( $\mathrm{n}=3$ spinal cords for each probe and each genotype). Limb muscle depletion in met ${ }^{d / d}$ embryos causes the death of all limb-innervating MNs, hence the complete loss of Pea3 ${ }^{+}$neurons and Ret-positive LMC but not axial columns. (C,D) Analysis of MN survival in Nestin-Cre (referred to as Nes) mediated neuronal-specificity-switch mutants, which display unaltered amounts of muscle. (C) High magnification views of ChAt ISH staining of the ventral horn of spinal cord cross-sections in Nes-Met ${ }^{\text {flox }+}$, Nes-Met ${ }^{d / f l o x}$, Nes-Met $^{2 P / f l o x}$, and Nes-Met ${ }^{2 S / f l o x}$ neonates (P2; $n=6$ for each group of animals) at C8-T1 level. In this region, MN clusters are organized in two groups (dashed lines). The more ventral cluster (red dashed lines) exhibits less MNs in the Nes-Met ${ }^{d / f l o x}$ mutant, in contrast to Nes-Met ${ }^{2 P / f l o x}$ and Nes-Met ${ }^{25 / f l o x}$ mutants, which are comparable to controls. (D) Quantification of MN numbers throughout the brachial region of Nes-Met ${ }^{d / f l o x}$, Nes-Met ${ }^{2 P / f l o x}$, and Nes-Met ${ }^{2 S / f l o x} \mathrm{P} 2$ mutants, showing percentages of MN loss per section. Positions of the C8, T1, and T2 DRGs are indicated by black lines. In the C8-T1 region, where percentages of MNs loss in Nes-Met ${ }^{d / f l o x}$ mutants are highest, Nes-Met ${ }^{2 P / f l o x}$ and Nes-Met ${ }^{25 / \text { flox }}$ mutants show non-significant MN loss, and are significantly different from Nes-met ${ }^{d / \text { flox }}$ mutants. Student's t test: ${ }^{*} P<0.05$, ${ }^{*} P<0.01$, ***P $<0.001$. Error bars represent standard error of the mean.

We have recently used conditional inactivation of met owing to Cre-mediated excision in the nervous system (Nestin-Cre) to demonstrate the direct control by HGF/ Met of the in vivo survival of a MN pool in the C8-T1 region corresponding to the pectoralis minor muscle [4]. As complete Nestin-Cre-mediated Met ablation occurred only after the onset of NOCD, this strategy allowed us not only to preserve muscle development, hence maintaining an unaltered amount of muscle in the limb, but also to bypass the early function of Met in the specification of the Pea3 pools and in motor nerve growth (Figure 6C,D) [4]. This genetic paradigm also allowed us to directly assess the net effect on MN survival attributed to Met-signalling changes in MNs in vivo. We therefore generated neuronal-specific-switch mutants in which one allele of met is floxed by loxP sites and the other allele carries the met signalling mutation: Nestin-Cre; $m e t^{d / f l o x}$, Nestin-Cre; met $^{2 P \text { flox }}$ and Nestin-Cre; met $^{2 S / f l o x}$ (referred to as Nes-Met $t^{d / f l o x}$, Nes-Met $t^{2 P / f l o x}$ and Nes-Met $t^{2 S / f l o x}$, respectively). In contrast to Nes-Met $t^{d / f l o x}$ mutants, the numbers of MNs measured in the Nes-Met ${ }^{2 P / f l o x}$ and Nes$M e t^{2 S / f l o x}$ mutants were equivalent to WT, with only minor non-significant reductions at $\mathrm{T} 1$ level (Figure 6C,D, Additional file 6: Figure S6). Thus, survival of the pectoralis minor $\mathrm{MN}$ pool is not compromised in neuralspecificity-switch Met mutants, as was found in our culture assays. Altogether, these results show that signalling routes downstream of the Met RTK can substitute for each other to promote MN survival and that those solicited by $\mathrm{Met}^{2 \mathrm{P}}$ and $\mathrm{Met}^{2 \mathrm{~S}}$ are equivalent in their efficacy in accomplishing this task. Such findings illustrate the remarkable degree of plasticity of Met signalling cascades ensuring survival, in contrast to the strict specificity required for Met-dependent axon guidance and to the partial flexibility for Pea3 identity acquisition by the HGF/ Met system.

\section{Discussion}

Signalling pathways are solicited to convey information from membrane receptors activated by growth factors to coordinate a set of outcomes including short-term transcription-independent responses and long-term geneexpression changes. When the same receptor is employed to trigger distinct biological responses in a given cell type, each response can be achieved through the activation of distinct signalling cascades. The degree to which a given signalling cascade is uniquely required for a specific biological response, or can be substituted by an alternative pathway, depends on the cell type, the nature of the response, the strength of the signal and the robustness of the signalling network available for this biological outcome. We have investigated this issue in vivo in the context of several biological requirements of the HGF/Met system in MNs, using a set of mouse lines carrying signalling mutations of Met that modulate the phosphotyrosine binding preferences, and thereby limit the range of signalling effectors mobilised by the modified receptor. We showed that in MNs, alternative HGF-induced Met cascades such as the PI3K or Src/Mek pathways, although biochemically or genetically distinguishable, are interchangeable to ensure MN survival, and MN specification, but not for axon guidance (Figure 7).

\section{Degree of equivalence of signalling cascades and robustness of a biological response}

A growing set of data supports the idea that signalling effectors are in charge of selective biological responses following RTK stimulation. While the view 15 years ago conveyed the idea that a given signalling cascade would be uniquely required for a given response, it has now emerged that several alternative pathways, although distinct from each other by their components, can substitute for one another to activate a given biological response. Therefore, the robustness of a biological outcome correlates with the capacity of cells not only to buffer variations of signal intensities [37], but also to offer a multiplicity of signalling molecules that can efficiently replace each other to ensure a given response [38,39]. Here we asked to which extent several biological responses to one pleiotropic RTK, Met, are compatible with substitutions of 


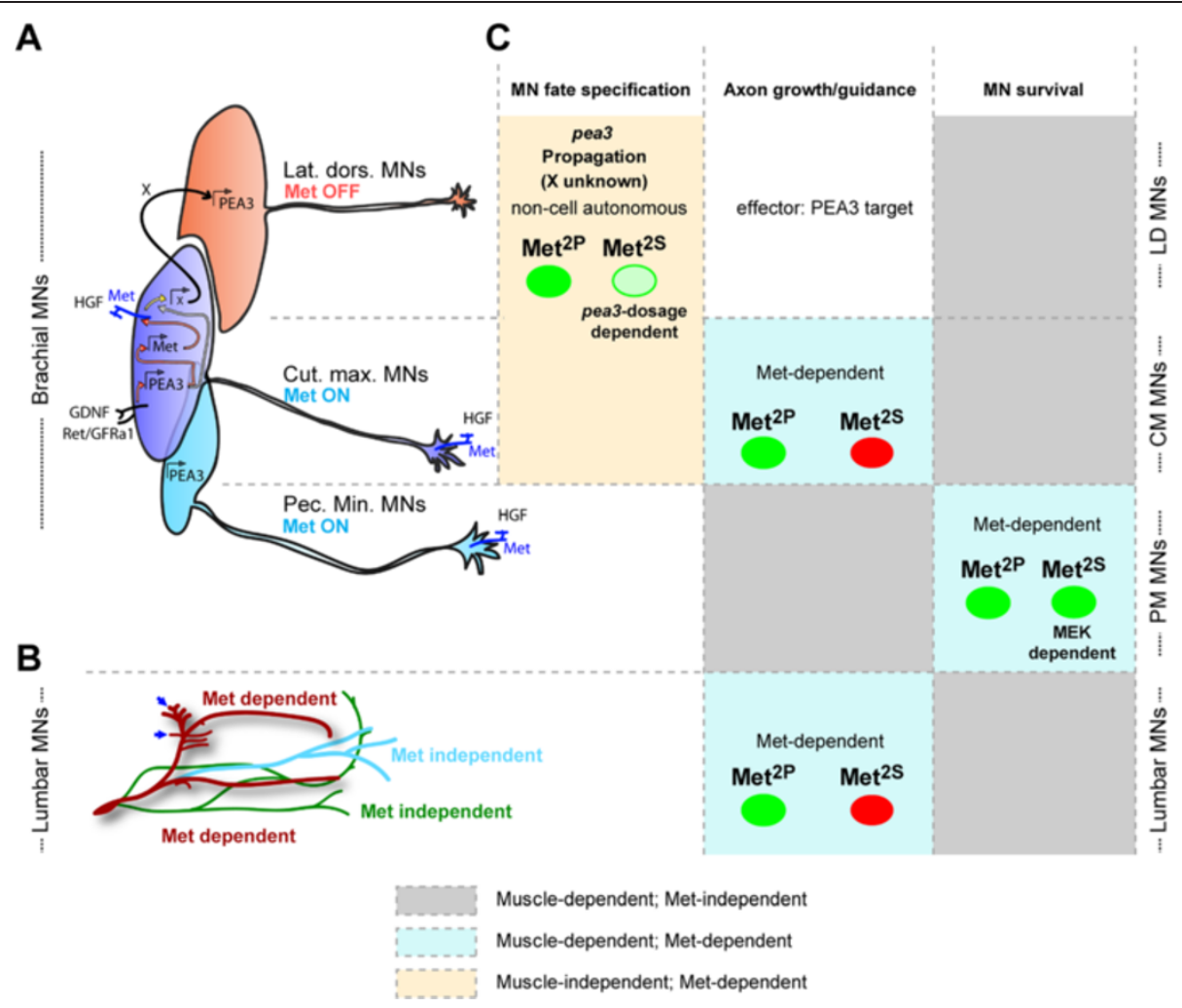

Figure 7 Summary of the signaling modalities characterizing each of the functions of HGF/Met in MNs. (A,B) Schematic summary of the MN populations in which HGF exerts its functions, highlighting which subsets express Met. (A) Scheme representing the three subsets of Pea3-expressing MNs, and the mechanism by which GDNF and HGF cooperate to establish Pea3 expression domain, based on [16]: GDNF acts on Ret/GFRa1 expressing "pioneer" neurons (dark blue pool, believed to largely match the CM MN pool in general), to induce Pea3 expression. Pea3 in turn is required to induce expression of the HGF receptor Met. HGF acts on the same "pioneer" neurons, once they express Met, to trigger the production by these neurons of a signal (referred to as X) that induces Pea3 expression in additional neurons ("recruited"). This leads to the lateral enlargement of the CM pool (within which Met expression propagates as well), and to the recruitment of more anterior neurons (red, largely matching the LD MN pool), which will not express Met. (B) Scheme representing lumbar axonal MN projections in the hindlimb, with colours distinguishing Met-dependent from Met-independent guidance. (C) Summary table of the various biological aspects of neuromuscular development involving HGF/Met. Columns distinguish the nature of the biological response (cell fate specification, axon growth/guidance, and survival); rows distinguish the MN populations, matching them to their position in the schemes in (A, top) and (B, bottom). The table indicates for each response/MN population the respective degree of muscle dependency, the corresponding function of HGF/Met, whether it is cell autonomous or not, and the degree of execution by signalling solicited by $\mathrm{Met}^{2 \mathrm{P}}$ and $\mathrm{Met}^{2 \mathrm{~S}}$ specificity-switch mutants (with green indicating complete execution, and red indicating abrogation).

signalling route downstream of the receptor for their faithful execution:

(a) Substitutable pathways, considered permissive for a given biological response, induce a cascade of biochemical events that converge towards and mobilise key activator(s) of this response, compatible with a positive outcome. Considered together, all components of alternative/substitutable pathways capable of mediating a given response can constitute an equivalence group, where the lack of one component (or lowered signalling levels by one component) can easily be compensated for by the network of available relationships between the others [39-41]. This concept has been instrumental in understanding pathways leading to cancer, where mutations in any component of a given equivalence group (also called a core pathway) are mutually exclusive and tend not to co-occur in the same tumour [41,42].

(b) Conversely, a pathway is considered non-permissive when it is not substitutable with another one, and when activation of an alternative pathway will not lead to the same output (even leading in some cases to the opposite outputs, for example attraction versus repulsion in axon guidance).

Whether certain pathways are equivalent (permissive) or not depends, on one hand, on the cell type (whether it is competent to offer a robust plasticity network to 
buffer signalling changes) and, on the other hand, on the nature of the biological outcome. Our results distinguish three types of biological outcomes, which are discussed in the following paragraphs:

\section{Non-equivalence of signalling cascades for axon branching towards muscle targets}

We showed that switching Met binding preferences towards Src family kinases precludes axonal branching to target muscles by HGF/Met, while accommodating PI3K recruitment ensures correct peroneal nerve patterning in the hindlimb (Figure 7). These differences of biological competences between $\mathrm{Met}^{2 \mathrm{P}}$ and $\mathrm{Met}^{2 \mathrm{~S}}$, with respect to axonal growth and intramuscular branching, are important for two main reasons:

(1) They allow unambiguous uncoupling of Met requirements for axonal growth from muscle development, and they also allow distinguishing of a nerve pattern defect from the consequences of an altered MN specification process (unlike for brachial Pea3 MN pools). The amount of muscle mass present in the limbs due to limited myoblast migration varies from embryo to embryo, although to a similar extent in $m e t^{2 P / 2 P}$ and $m e t^{2 S / 2 S}$ embryos (Figure 2C,D). Cases with no muscles (where the $T A$ muscle is completely missing) result in the absence of nerve branches exiting the main peroneal nerve, even in a small subset of met $^{2 P / 2 P}$ embryos. This reflects the fact that muscles are necessary for sending a branching signal. However, in the presence of equivalent amounts of muscle mass, such branches are unequivocally detected in $m e t^{2 P / 2 P}$ but not in $m e t^{2 S / 2 S}$ embryos, where the nerve pattern is indistinguishable from that seen in $m e t^{d / d}$ mutants. This indicates that Met signalling is essential for the decision of motor axons to exit the main nerve and enter the muscle, provided that the competent signalling effectors are mobilised. According to these results, the muscle-derived signal may be either HGF itself or a protein necessary for HGF to bind/activate its receptor, such as enzymes involved in HGF precursor cleavage and/or in its extracellular diffusion [43-45].

(2) The complete inability of the $\mathrm{Met}^{2 \mathrm{~S}}$ receptor to execute an axonal branching decision, which contrasts with its capability to trigger MN survival, indicates that Src family kinase activation is inadequate for axonal branching by HGF/Met (or that Src antagonises the response otherwise mediated through another pathway such as PI3K; see below). These results apparently contrast with other ligand/receptor systems in which Src family kinases have been reported to be required for axon guidance but not for survival of MNs [46]. In this study, interfering with Src signalling in developing chicken spinal cords resulted in axonal path-finding errors at the dorsoventral choice point in the limb [46], a decision cooperatively controlled by ephrinA/EphA and GDNF/Ret signalling pathways [16,47-49]. Similarly, inactivation of the Drosophila Src homologue causes aberrant midline crossing indicating that $\mathrm{Src}$ is required to inhibit crossing of longitudinal axons [50]. A possible explanation for this apparent discrepancy with our results may be that Src family kinases are involved in non-permissive pathways, where they instruct axons of the behaviour they should adopt (whether they should be attracted or repelled) in response to various signalling cues. Consistently with this hypothesis, Src family kinases are involved in repulsive responses downstream of ephrin reverse signalling [51,52], but mediate attractive turning responses to Netrin [53]. Thus, for the HGF/Met system, Src activation by $\mathrm{Met}^{2 \mathrm{~S}}$ may have turned an attractive/positive response into a negative/repulsive one, for example by mobilising a cascade normally involved in a negative response to another signal. In such a context, Src activation would therefore not be dispensable, but rather would antagonise the normal function of Met. In contrast, the PI3K pathway appears as a permissive pathway, which may serve as a switch to activate responsiveness or as a rheostat to modulate the intensity of a unique one-sided response of axonal growth [54]. What determines whether Src family kinases will mediate an attractive or repulsive response may be encoded by intrinsic differences between neuron subtypes, for example through differential expression of receptor complexes or signalling effectors involved in gating the nature of this decision [55-57]. An alternative possibility could be that in the WT context, Src family kinase recruitment by Met occurs only in a MN subtype-specific manner; for example Src may not be solicited in those MNs in which Met regulates guidance (CM, peroneal axons). In this context, modifications of the Met binding preference may result in an ectopic Src family kinase activation, thereby removing the pool-specificity and silencing the response of neurons.

\section{Two non-equivalent pathways can substitute for each other to control cell fate acquisition}

In contrast to the clear incapacity of signalling by the $\mathrm{Met}^{2 \mathrm{~S}}$ receptor to execute axon guidance decisions, the cascade of events allowing expansion of the Pea3 expression domain in the brachial motor columns is efficiently executed by both $\mathrm{Met}^{2 \mathrm{P}}$ and $\mathrm{Met}^{2 \mathrm{~S}}$ receptors, the two 
pathways being, however, differentially sensitive to Pea3 gene dosage (Figure 7). Pea3 expression was previously shown to be initially activated by GDNF in a subset of brachial MNs [31]. We have previously added another degree of complexity by showing that this induction was restricted to a pioneer group of Met-expressing neurons, in which Met is required to trigger the production of a signal propagating Pea3 expression to anterior neurons (Figure 7A, Additional file 3: Figure S3 and [16]). With known inducers of Pea3, such as FGF8/3 and Wnt1, not being expressed in the pioneer pool, work is currently underway to identify the signal, induced by HGF/Met in pioneer neurons, that is responsible for expansion of the Pea3 expression domain, not only laterally, within the Met-expressing CM MN pool, but also to Met-negative anterior MNs. The results of the present study indicate that the signalling cascades mobilised by both $\mathrm{Met}^{2 \mathrm{P}}$ and $\mathrm{Met}^{2 \mathrm{~S}}$ receptors equivalently allow triggering of the production by pioneer neurons of this signal mediating expansion of Pea3 expression to recruited neurons. The sensitivity to lowered Pea3 levels in the $\mathrm{Met}^{2 \mathrm{~S}}$ scenario indicates that the responsible signalling cascade involves Pea3 transcriptional activity. This further implies that this Pea3-inducing signal must be a Pea3 target gene. The possibility that Pea3 could be involved in its own propagation is consistent with the reduced domain of Pea3-lacZ expression described for Pea3-null embryos [32] and with the fact that Met expression in the pioneer domain is regulated by Pea3 (Additional file 1: Figure S1) [16]. The differential effectiveness of the signalling cascade activated by the $\mathrm{Met}^{2 \mathrm{P}}$ and $\mathrm{Met}^{2 \mathrm{~S}}$ receptors suggests that Pea3 transcriptional activity might be regulated by Src but not by PI3K. Pea3-family transcription factors are typically known to be activated by a Ras-Mek axis downstream of FGF signalling [58-62]. A recent study shows that ERK signalling mediates induction of Etv4 (pea3) and Etv5 expression in sensory neurons by target-derived neurotrophic factors such as NGF [63]. Our own result from MN survival assays suggest that the pathway mobilised by the $\mathrm{Met}^{2 \mathrm{~S}}$ receptor for survival also involved the ERK pathway through Mek activation. It is therefore tempting to speculate the existence of a Src-Mek-Pea3 circuit available downstream of HGF/Met in MNs. Consistent with this hypothesis, Pea3 was shown to be involved downstream of $\mathrm{v}$-src signalling to activate transcription of collagenase 1/MMP1 [64].

Moreover, Pea3 loss-of-function disrupts Src activation and cell mobility in Pea3-null fibroblasts, indicating that Src can also be activated and required downstream of Pea3 [65]. Such a reciprocal relationship between Pea3 and Src could explain why a twofold lowering of Pea3 expression does not impair propagation of Pea3 expression in a WT Met signalling context, but becomes critical in the context of a signalling-switch mutant of Met predominantly relying on Src activation. In contrast, the fact that signalling by the Met ${ }^{2 \mathrm{P}}$ receptor is not sensitive to reduced Pea3 dosage indicates that production of the signal mediation expansion of Pea3 expression can also be induced by other transcription factor(s). Thus, the robustness of the cell fate specification function triggered by the Met RTK relies on a complex signalling circuit possibly composed of a combination of distinct transcription factors, the activity of which is modulated qualitatively and/or quantitatively by upstream signalling cascades.

\section{Equivalence of signalling cascades for neuronal survival}

Finally, we found that signalling pathways mobilised by either the $\mathrm{Met}^{2 \mathrm{P}}$ or $\mathrm{Met}^{2 \mathrm{~S}}$ receptors were equally capable of substituting for the WT pathways in regulating MN survival. This is supported by primary MN culture experiments and by in vivo studies on Met-dependent pectoralis minor MN pool survival [4] using neuronalspecificity-switch Met mutants. In vitro, survival by HGF in $m e t^{2 \mathrm{P} / 2 \mathrm{P}} \mathrm{MNs}$ is selectively mediated by PI3K, but not by $\mathrm{Src}$ or Mek, whereas in $m e t^{2 \mathrm{~S} / 2 \mathrm{~S}} \mathrm{MNs}$, survival requires Src and Mek, but not PI3K. Neuronal survival by numerous neurotrophic factors, including HGF in MNs [35], involves the traditional PI3K-Akt pathway. Akt regulates components of the apoptotic machinery, such as Bad, a pro-apoptotic member of the Bcl-2 family, and Forkhead box transcription factors, which are involved in expression of other death genes [66,67]. Besides this main road, alternative pathways can also be employed for cell survival [68]. For example, a number of studies have provided evidence that Src is required for survival promoted by GDNF, by cytokines, such as CNTF, or by the P2Y2 receptor, in cultured embryonic MNs or osteoclasts. Nevertheless, in these cell types Src survival properties are still dependent on the PI3K-Akt pathway [10,35,69-71]. The requirement of Src and Mek, but not PI3K, for MN survival by $\mathrm{Met}^{2 \mathrm{~S}}$ implies either a parallel action of Src and Mek-ERKs pathways, or the existence of a circuit involving activation of Mek-ERKs by Src. A previous report on chick MNs has indicated that Mek signalling is dispensable for their survival [36]. However, saturating concentrations of HGF $(10 \mathrm{ng} / \mathrm{ml})$ were used in this study, whereas we favoured assessing the signalling required for survival using HGF doses more similar to the physiological context $(0.4 \mathrm{ng} / \mathrm{ml}$ or $2 \mathrm{ng} / \mathrm{ml})$. It is possible that with saturating amounts of ligand, pathways such as PI3K are over-activated to an extent that is sufficient for MN survival, even in the absent of other signalling components such as Mek. In contrast, with the lower concentration of HGF that we used (closer to the levels occurring in vivo), the level of activation of each individual pathway may not be sufficient to promote survival independently of the others. This scenario 
coincides with the signalling requirements for survival we have evidenced in vivo, in the context of our neuronalrestricted Met specificity-switch mutants.

Intriguingly, the signalling pathways initiated by $\mathrm{Met}^{2 \mathrm{P}}$ and $\mathrm{Met}^{2 \mathrm{~S}}$ receptors are adequate for survival of MNs, but not of embryonic hepatocytes both in vitro and in vivo [23-25]. Such a paradox illustrates the extent to which the signalling efficacy for a given biological response is influenced by the cell type. This may be related to intrinsic factors, such as the biological state of cells (e.g. proliferating versus quiescent), or to extrinsic factors, such as extracellular levels of stress signals. Another reason may be differences in expression patterns and levels of signalling components in embryonic MNs versus hepatocytes. In this respect, Additional file 7: Figure S7 offers an illustration of the expression patterns of several members of various signalling families, based on the GenePaint database [72]. It is interesting to note that several adaptors expressed at high levels in the nervous system, some of them even specifically in brachial MN pools, appear either not to be expressed in the liver or to be expressed at much lower levels.

The high plasticity of signalling networks controlling MN survival is particularly intriguing, when taking into account the unexpected discovery that PI3K, Src and Mek-ERK pathways are all necessary downstream of the WT Met receptor, each of them being, however, insufficient on its own to provide a robust survival response in the absence of the other pathways in vitro. This contrasts with the fact that Src is dispensable downstream of $\mathrm{Met}^{2 \mathrm{P}}$ and PI3K is dispensable downstream of $\mathrm{Met}^{2 \mathrm{~S}}$. A possible explanation of this apparent discrepancy could be that in MNs, Met specificity-switch receptors behave as mild gain-of-function mutants for some pathways, while being incapable of activating the others. This increased level of activation of individual signalling routes downstream of $\mathrm{Met}^{2 \mathrm{P}}$ or $\mathrm{Met}^{2 \mathrm{~S}}$ would raise their activity above a threshold necessary to activate effectors of survival, whereas this threshold is only reached by the combined activity of PI3K, Src and MAPK pathways downstream of WT Met. According to this scenario, such increased levels above the threshold could alleviate the simultaneous requirement for the other pathways to induce a survival response successfully in MNs. As mentioned above, this contrasts with hepatocytes, in which signalling initiated by $\mathrm{Met}^{2 \mathrm{P}}$ or $\mathrm{Met}^{2 \mathrm{~S}}$ receptors is insufficient to ensure a survival response to HGF [23-25], thus indicating that the gain-of-function character is conditioned by the competence of the cell type. Molecular analysis of signalling circuits downstream of Met WT and Met mutants would offer the possibility of uncovering the components and levels of their activation that ensure the robustness of survival circuits in MNs. However, addressing this question would require detailed molecular and biochemical analyses that are technically not compatible with the number of MNs accessible from embryos, and would be challenged by the switch in biological responses and in MN subtypes occurring throughout development.

\section{Conclusions}

How can we interpret the specificity of signalling pathways necessary for axonal growth compared to the plasticity allowed when executing RTK-induced MN survival or specification responses? Axon guidance is a complex example of a biological response regulated by several non-equivalent signalling components. Their activation leads to choices between multiple qualitatively different outcomes, such as stop, go, turn, exit, etc., thereby requiring intercalation of multiple signalling systems to gate the decision-making process at the level of the growth cone. Such a context implies that some signalling cascades need to be specifically associated with selective outcomes rather than being permissive, for the proper integration of multiple instructive axon guidance signals. In contrast, MN survival and specification are dichotomic choices at the cell level, integration of which at the tissue level involves the quantitative assessment of individual neuron responses to the limited amount of neurotrophic factors produced by target tissues. Adaptation of the MN numbers in each pool to the size of its cognate muscle target is achieved by a mechanism capable of measuring accurately the amount of target-derived signal available, and of adjusting this amount with respect to an intrinsic threshold within each cell, to block the apoptotic cascade or to activate the specification program. Likewise, raising RTK signalling levels above endogenous thresholds can enhance the neuroprotective effect and limit susceptibility to $\mathrm{MN}$ diseases [73]. The plasticity allowing several alternative pathways to substitute for each other to achieve such responses confers robustness to the mechanisms coordinating the development and size of the neuromuscular system.

\section{Methods}

\section{Animals and genotype analysis}

The generation of the different signalling alleles of the met gene used in this study $\left(m e t^{d}, m^{2 P} t^{2}\right.$ and $m e t^{2 S}$ ) has been described previously $[9,15]$. The original neo ${ }^{+}$alleles, which contained a neo selection cassette flanked by LoxP sites, were referred to as met ${ }^{2 \mathrm{Pneo}}$ and met $^{2 \mathrm{Sneo}}$. Alleles without neo were obtained after excision of the neo cassette by crossing mutant mice with the Deleter-Cre transgenics [9]. Genotype analysis by PCR was performed as described $[9,12]$. The met ${ }^{\text {LacZ }}$ allele is an alternative knockout/knock-in allele of met in which the LacZ reporter gene also reflects expression of the endogenous met gene [4]. In the original allele, $L a c Z$ expression is conditioned by the removal of a Lox-stop-Lox cassette [4]. In 
the allele used here, by crossing with a deleter-cre line, we have derived a mouse line in which the stop cassette has been permanently deleted. Genotype analysis was performed by PCR as described [4]. Pea3-lacZ mice were used with the permission of Arber and Jessell, and genotyped as previously described [32]. Animals were maintained and sacrificed in accordance with institutional guidelines. Adult mice were euthanised by cervical dislocation.

\section{In situ hybridisation}

Embryos were collected in phosphate-buffered saline (PBS) and fixed in 4\% paraformaldehyde (PFA). ISHs were performed with the relevant RNA probe on either whole-mount embryos, dissected limbs or spinal cords, according to previously published procedures [16]. Whole spinal cord ISH was performed as described [74], with digoxigenin-labelled RNA probes for met, MyoD (obtained from Ponzetto), Pea3, (from Jessell), Ret (from Rosenthal) and ChAT (choline acetyl transferase; from Henderson). Spinal cords were flat mounted as open-book preparations with MNs on the upper side, and imaged using a Zeiss Axiophot (Marly le Roi, France) or Leica stereomicroscope (Wetzlar, Germany). Whole-mount embryos were partially cleared in 50\% glycerol and imaged using a stereomicroscope.

$X$-Gal and Salmon-Gal staining for $\beta$-galactosidase activity Staining for $\beta$-galactosidase activity was performed using two alternative methods. For spinal cords of embryos carrying the pea $3^{\text {Lac } Z}$ allele, we employed the traditional protocol using X-Gal substrate in combination with ferric and ferrous ions. Briefly, spinal cords were dissected from freshly collected embryos, fixed in 4\% PFA in PBS (40 min), rinsed in PBS, and incubated in X-Gal in combination with potassium ferricyanide and ferrocyanide $(\mathrm{FeCN})$. To detect $\beta$-galactosidase in the spinal cords of embryos carrying the met ${ }^{\mathrm{LacZ}}$ allele, we used an alternative method based on Salmon-Gal (6-chloro-3-indolyl-beta-Dgalactopyranoside from Appolo scientific, Maschester, UK) in combination with tetrazolium salt [75]. Briefly, spinal cords were dissected from freshly collected embryos, fixed in $1 \%$ PFA and $0.2 \%$ glutaraldehyde in PBS (10 min), rinsed three times in PBS, then incubated for a minimum of $7 \mathrm{~h}$ overnight at $37^{\circ} \mathrm{C}$ in pre-stain solution without substrate (potassium ferricyanide $200 \mathrm{mM}$, potassium ferrocyanide $200 \mathrm{mM}, 4 \mathrm{mM} \mathrm{MgCl} 2$ and $0.04 \% \mathrm{NP} 40$ in PBS) to reduce endogenous $\beta$-galactosidase activity. Samples were then rinsed three times in PBS, and incubated in staining solution (1× PBS, with $0.04 \% \mathrm{NP} 40,2 \mathrm{mM} \mathrm{MgCl}_{2}, 1 \mathrm{mg} / \mathrm{ml}$ Salmon-Gal (stock solution $40 \mathrm{mg} / \mathrm{ml}$ in dimethyl sulfoxide (DMSO)) and $0.33 \mathrm{mg} / \mathrm{ml} \mathrm{NBT}$ (stock solution $75 \mathrm{mg} / \mathrm{ml}$ in $70 \% \mathrm{DMF})$ ), for about 30 to $40 \mathrm{~min}$ at $37^{\circ} \mathrm{C}$. In all cases (X-Gal and Salmon-Gal), staining was terminated by rinsing in PBS, and post-fixing in 4\% PFA. Spinal cords were flat mounted in 1 volume glycerol/1 volume $4 \%$ PFA for imaging.

\section{Embryonic motor neuron cultures}

E12.5 mouse embryos were collected in Hibernate (E) medium (Life Technology) with B-27 supplement (Life Technology) and kept on ice until the dissection, while the genotype was determined by PCR. Ventral spinal cords from an equal number of WT and mutant embryos were dissected. MNs were isolated from either brachial + lumbar, or from cervical + thoracic + sacral segments, by a previously described method [34] involving a combination of centrifugation on BSA cushions and a metrizamide density gradient centrifugation. At the end of the procedure, the cell suspension was highly enriched in MNs. For survival assays, MNs were plated in polyornithine/ laminine-coated four-well tissue culture dishes $(1,500$ neurons per well) in Neurobasal medium (Life Technology, Saint Aubain 91190, France). Recombinant neurotrophic factors and inhibitors were added $2 \mathrm{~h}$ after seeding. MNs were kept for 3 days either in Neurobasal medium or in the presence of GDNF or HGF (both obtained from R\&D, Minneapolis, MN, USA). All inhibitors (LY294002, PD98059, PP2 and PP3) were obtained from Calbiochem (Darmstadt, Germany). PP3 was used as a negative control for PP2 (not shown). MN survival was quantified by counting large bright unipolar neurons with long axonal processes on the whole area of each well.

\section{Whole-mount anti-neurofilament immunohistochemistry}

Embryos were collected in cold PBS, fixed for $2 \mathrm{~h}$ in $4 \%$ PFA in PBS, and post-fixed overnight in Dent's fixative (80\% methanol, $20 \% \mathrm{DMSO}$ ) at $4^{\circ} \mathrm{C}$. Whole-mount antineurofilament immunohistochemistry was performed as previously described [29]. Briefly, embryos were bleached for $4 \mathrm{~h}$ in $6 \% \mathrm{H}_{2} \mathrm{O}_{2}$ in Dent's solution and further rehydrated through a progressive methanol series. Antibody incubations were performed in $80 \%$ newborn calf serum, $20 \%$ DMSO, $0.5 \%$ triton with thimerosal. Antibodies used were the following: anti-neurofilament antibody $(2 \mathrm{H} 3$ and DSHB) and HRP-conjugated goat anti-mouse antibody (Sigma). Staining was developed using DAB Tablets (Sigma, Saint-Quentin Fallavier, France). Embryos were cleared for imaging in BABB (1:2, benzyl alcohol/benzyl benzoate).

\section{Image processing and analyses}

Quantification of signal intensity was performed using the ImageJ software. Briefly, images of ISH were first converted to a grey scale and inverted to a negative scale (with the highest signal intensity matching being white and the lowest black). Signal intensity was measured along a horizontal line of a given pixel length (matching half a flatmounted spinal cord, always positioned where indicated 
with dotted lines on Figure 3). After background and threshold subtraction, the values were averaged between several samples of each genotype (considering left and right sides separately, for the number of spinal cord sides used, for each plot indicated in the corresponding figures) to generate an average signal distribution plot. The total signal intensity was also calculated for each sample, and plotted individually (Figures 2 and 5, Additional file 1: Figure S1 and Additional file 4: Figure S4).

\section{Statistical analysis}

Results were expressed as mean \pm standard error of the mean. Statistically significant differences were assessed by unpaired Student's $t$-test when data were showing a normal distribution, and by the Mann-Whitney test otherwise. * indicates $P<0.05$ and ** indicates $P<0.001$.

\section{Additional files}

Additional file 1: Figure S1. Figure S1. Met-deficiency-triggered reduced limb muscle volume does not cause MN loss at E12.5. (A) Analysis of muscle migration by whole mount MyoD ISH on E11.5 (top row) or E12.5 (bottom row) embryos, showing forelimbs Of WT and met signalling mutants ( $n=3$ embryos per genotype and stage). (B) Ret ISH in brachial spinal cords from E12.5 WT, met ${ }^{d / d}$, met $^{2 P / 2 P}$, met ${ }^{2 / 2 S}$ embryos. (C) The size of the Ret-expressing brachial MN pools was assessed using measurements with Image J of Ret ISH staining intensity along the orange dotted line at C7 level. Each plot represents the average signal distribution of the indicated number of spinal cord sides $\left(m e t^{+/+}: n=18\right.$; met $^{d / d}: n=14 ;$ met $^{2 P / 2 P}: n=10$; and met $^{2 S / 2 S}: n=8$ ). (D) Met expression was

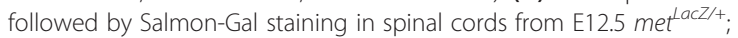

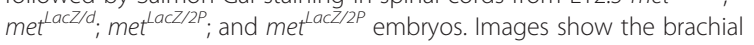
region of the same spinal cords shown in Figure 3C. (E) Quantification of the size of the Met-expressing brachial neurons along the orange line at C7 level. Each plot represents the average signal distribution of the indicated number of spinal cord sides (met ${ }^{\text {acZ//+ }}: \mathrm{n}=22$; met $^{\text {LacZ/d}}: \mathrm{n}=8$; met $^{\operatorname{Lac} Z / 2 P_{2}}: \mathrm{n}=18$; and met $\left.{ }^{\operatorname{Lac}_{2} / 2 P}: \mathrm{n}=10\right)$. $(\mathbf{F}, \mathbf{G})$ Quantifications and statistical analyses of the sum of signal intensity corresponding to measurements of Ret ISH staining (F), or Met ${ }^{\text {LacZ }}$ expression (G), based on intensity plots in $(\mathbf{C})$ or $(\mathbf{E})$, respectively. Numbers of samples are as indicated in (C) and (E). At E12.5, the size of the Ret-expressing MN population is not significantly altered in met mutant embryos, as MN numbers are independent of muscle mass at this stage. In contrast, analysis of met ${ }^{\text {Lacz }}$ expression confirms that met is required for its own expression in the C7-C8 brachial pool, signalling via either PI3K or Src being equally efficient to ensure establishment of met expression domain.

Additional file 2: Figure S2. Altered peroneal nerve guidance in absence of Met signalling cannot be attributed to lowered Pea3 expression. (A) Scheme of the lumbar spinal nerves and plexuses at hindlimb levels (same as in Figure 3A) based on a lateral view of an E11.5 WT embryo, showing that the sacral plexus is located caudal to the sciatic plexus, and collects axons mostly originating from L6 and S1 spinal segments. (B) Double ISH with Met (blue) and Pea3 (red) probes (left), or with the Pea3 probe only (right), was performed for E12.5 WT spinal cords to show their respective expression patterns in the lumbar region in apposition with spinal segments and the corresponding plexuses shown in (A). Overall a longitudinal column expressing Pea3 lies more ventral (closer to the midline) than the Met-expressing motor neurons at L4-5-6 levels, while the caudal part of the Pea3 domain maps to the sacral segments, thus corresponding to projections to the sacral plexus. Therefore, Pea3 is not expressed in Met-expressing peroneal motor neurons (yellow dotted pools). (C) Analysis of Pea3 and its target gene
Sema3E expression in lumbar spinal cords of WT and met ${ }^{d / d}$ embryos confirms that, as previously shown for the brachial region, Met is also required for expansion of expression of Pea3 and Sema3E in the lumbosacral region ( $n=4$ spinal cords for each probe and each genotype). (D) Anti-neurofilament (NF) immunohistochemistry for hindlimbs of WT and Pea3 ${ }^{\text {LacZ/LacZ }}$ E12.5 embryos, showing that Pea3 functions are dispensable for peroneal nerve guidance. (E) High magnification of the area from a WT E13.5 hindlimb stained with anti-NF antibody, where side branches exit the deep peroneal nerve to make contacts with muscles. The right panel shows the same picture a white line highlighting the nerve, and green lines highlighting side branches.

Additional file 3: Figure S3. Scheme representing the three subsets of Pea3-expressing MNs, and the mechanism by which GDNF and HGF cooperate to establish Pea3 expression domain, based on [16]: Step1: prior to GDNF secretion by the plexus mesenchyme, Pea3 is not expressed, but a subset of neurons express the receptor Ret/GFRa1 coupe. Step2: GDNF acts on Ret/GFRa1 expressing "pioneer" neurons (dark blue pool, believed to largely match the CM MN pool), to induce Pea3 expression. Pea3 in turn is required to induce expression of the HGF receptor Met. Step3: HGF acts on the same "pioneer" neurons, once they express Met, to trigger the production by these neurons of a signal (referred to as $X$ ) that induces Pea3 expression in additional neurons ("recruited"). This leads to the lateral enlargement of the CM pool (within which Met expression propagates as well), and to the recruitment of more anterior neurons (red, largely matching the LD MN pool), which will not express Met. Expression of Pea3 in the Pec Min MN pool appears to occur independent of GDNF [31], but which peripheral factor is required for this induction has not been identified.

Additional file 4: Figure S4. MN survival is proportional to the amount of muscle in developing limbs in embryos with attenuated expression levels of Met-signalling variants. (A) Analysis of muscle migration by whole-mount MyoD ISH in WT, met $^{2 \mathrm{Pn} / 2 \mathrm{Pn}}$ and $\mathrm{met}^{2 \mathrm{Sn} / 2 \mathrm{Sn}}$ E12.5 embryos at forelimb levels ( $n=3$ embryos for each genotype). Note that the presence of a neo cassette in the met locus further reduces the ability of mutant receptors to promote myoblast migration and colonisation of the limb, due to attenuation of receptor expression levels. Consequently, muscle masses in $\mathrm{met}^{2 P n / 2 P n}$ and met $^{2 S n / 2 S n}$ embryos are reduced compared to met $^{2 P / 2 P}$ and met $^{2 S / 2 S}$ embryos (see Additional file 1: Figure S1A,B). (B-E) ISH was performed with Pea3 $(\mathbf{B}, \mathbf{D})$ and $\operatorname{Ret}(\mathbf{C}, \mathbf{E})$ RNA probes on spinal cords from E12.5 (B,C) and E14.5 (D,E) WT, met $^{2 P n / 2 P n}$ and met $^{2 S n / 2 S n}$ embryos (with a minimum $n=3$ spinal cords for each probe, stage and genotype). Quantification of the lateral expansion of the Pea3 domain (B) and assessment of MN numbers through Ret ISH (C), were performed by measuring signal intensity; each plot represents the average signal distribution measured on the indicated number of spinal cord sides (WT $n=14$ for Pea3 and $n=18$ for Ret; met $^{2 P n / 2 P n} n=6$ for each probe; met $^{2 S n / 2 S n} n=6$ for each probe), the two sides being considered separately, along the red dotted line for each image. Measurements were performed with ImageJ, after negative conversion and background subtraction. This analysis shows that prior to muscle dependency, overall MN numbers are unaffected by reduced muscle mass and that the $\mathrm{Met}^{2 \mathrm{P}}$ and $\mathrm{Met}^{2 \mathrm{~S}}$ receptors, in spite of reduced met expression levels in met ${ }^{2 P n / 2 P n}$ and met $^{2 S n / 2 S n}$ embryos, retain an intermediate but comparable capacity to promote expansion of the Pea3 expression domain (as quantified in (C), $P>0.89$ ). (D,E) After the onset of muscle dependency (at E14.5), as expected, the reduced MN survival in met ${ }^{2 P n / 2 P n}$ and met $^{25 n / 2 S n}$ embryos correlates with reduced muscle masses. Again, $m_{e t^{2 P n / 2 P n}}$ and met $^{25 \mathrm{n} / 2 S \mathrm{Sn}}$ embryos do not appear different from each other.

Additional file 5: Figure S5. Detection of Met-expressing MNs in the brachial spinal cord of Met signalling mutants. Met expression was detected in E13.0 spinal cords from WT, met ${ }^{d / d}$, met ${ }^{2 P / 2 P}$ and $m e t^{25 / 2 S}$ embryos (minimum $n=3$ spinal cords per genotype) by ISH with an RNA probe matching the met extracellular domain, which was unchanged by the genetic modifications to the met locus. The spinal cords (unopened) are imaged from their left side, at the brachial level, with anterior to the left and posterior to the right.

Additional file 6: Figure S6. MN numbers in neural-specific Met signalling mutants. MN numbers were quantified at P2 in the brachial region $(\mathrm{C} 8-\mathrm{T} 1)$ of Nes-Met ${ }^{2 \text { Pflox }}(n=8)(\mathbf{A})$ and Nes-Met ${ }^{2 \text { S/flox }}(n=7)$ 
(B) mutants compared to controls ( $m e e^{\text {flox/+ }} n=4$ ). ChAT-expressing MNs were quantified on cryosections shown in Figure 5C. Positions of the C8, T1 and T2 DRGs are indicated by black lines. These graphs correspond to the graphs shown in Figure 5D displaying percentage of loss.

Additional file 7: Figure S7. Potential for plasticity of signalling pathways illustrated through expression patterns of signalling effectors. Sagittal sections of E14.5 mouse embryos hybridised with the indicated probe (expression data are extracted from the GenePaint database). Brachial MN pools are indicated by arrows. (A) Src family kinases and associated effectors. (B) PI3K subunits and downstream effectors (Akt1). (C) MAPK family and effectors. When the indicated gene is expressed in brachial MN pools, a magnification of the area is shown.

\section{Abbreviations}

BSA: Bovine Serum Albumine; CM: Cutaneous maximus; cut.pn: cutaneous (sensory) peroneal; d.pn: deep peroneal; dm: distal muscle mass (un-split dorsal hand muscles); DMF: Dimethylformamide; DMSO: Dimethyl-sulfoxide; DRG: Dorsal Root Ganglion; EDL: Extensor digitorum longus; edl: extensor digitorum longus; edl.b: branches entering the extensor digitorum longus muscle; Etv4: Ets variant gene 4 (formerly called Pea3 for polyomavirus enhancer activator 3); GDNF: Glial-derived Neurotrophic Factor; HGF: Hepatocyte Growth Factor; ISH: in situ hybridization; LD: Latissimus dorsi; LMC: Lateral Motor Column; MN: Motor Neuron; Muscles: ta: tibialis anterior; NBT: Nitro Blue Tetrazolium; neo: Neomycin resistance gene; Nerves: co.pn: common peroneal; PBS: Phosphate-buffered saline; PCR: Polymerase chain reaction; pd: peroneus digiti (includes peroneus digiti quarti and peroneus digiti quinti); PFA: Paraformaldehyde; PI3K: Phosphatidylinositol 3-kinase; pl: peroneus longus; PTB: Phosphotyrosine Binding Domain; s.pn: superficial peroneal; SH2: Src homology domain type 2; SH3: Src homology domain type 3; TA: Tibialis anterior; ta.b: branches entering the tibialis anterior muscle; WT: Wild-type.

\section{Competing interests}

The authors declare that they have no competing interests.

\section{Authors' contributions}

FM and FH are co-senior authors. FH and FM conceived, designed and directed the study, and analysed and interpreted the data. FH, NC, BH, FL and VA-G performed the experiments. FH wrote the manuscript with proofreading by FM. Final approval was given by all authors.

\section{Acknowledgements}

We thank Chris Henderson for invaluable intellectual support; Ruediger Klein for supporting this study in its early stages and for valuable comments on the manuscript, and former members of his laboratory for insightful discussion; Elise Arbeille for help with ISHs of spinal cord sections; Sylvie Richelme for technical help; Virginia Girod-David and Leo Jullien for excellent help with mouse husbandry at the IBDM; and Angela Zimmermann for comments on the manuscript. Work in the Helmbacher laboratory was supported by funds from the CNRS (ATIP grant), FRM (Fondation pour la Recherche Médicale, Equipe FRM 2007), AFM (Association Française contre les Myopathies), FdF (Fondation de France), ARC (Association pour la Recherche contre le Cancer) and ANR (Agence Nationale de la Recherche, ANR Neuro 2007). Work in the Maina lab by FM was supported by FRM, AFM, FdF, ARC, Fondation Bettencourt-Schueller and INCa (Institut National du Cancer). The contributions of the Region Provence Alpes Côtes d'Azur and Aix-Marseille Université for the IBDM animal facility, and of the France-Biolmaging/PICSL infrastructure (ANR-10-INSB-04-01) for the imaging facility are also acknowledged.

\section{Received: 1 July 2014 Accepted: 4 July 2014}

\section{Published online: 14 August 2014}

\section{References}

1. Kanning KC, Kaplan A, Henderson CE: Motor neuron diversity in development and disease. Annu Rev Neurosci 2010, 33:409-440.

2. Dasen JS: Transcriptional networks in the early development of sensory-motor circuits. Curr Top Dev Biol 2009, 87:119-148.

3. Polleux F, Ince-Dunn G, Ghosh A: Transcriptional regulation of vertebrate axon guidance and synapse formation. Nat Rev Neurosci 2007, 8:331-340.
4. Lamballe F, Genestine M, Caruso N, Arce V, Richelme S, Helmbacher F, Maina F: Pool-specific regulation of motor neuron survival by neurotrophic support. J Neurosci 2011, 31:11144-11158.

5. Shneider NA, Brown MN, Smith CA, Pickel J, Alvarez FJ: Gamma motor neurons express distinct genetic markers at birth and require muscle spindle-derived GDNF for postnatal survival. Neural Dev 2009, 4:42.

6. Pawson T, Scott JD: Signaling through scaffold, anchoring, and adaptor proteins. Science 1997, 278:2075-2080.

7. Medina DL, Sciarretta C, Calella AM, Von Bohlen Und Halbach O, Unsicker K, Minichiello L: TrkB regulates neocortex formation through the Shc/PLCYmediated control of neuronal migration. EMBO J 2004, 23:3803-3814.

8. Postigo A, Calella AM, Fritzsch B, Knipper M, Katz D, Eilers A, Schimmang T, Lewin GR, Klein R, Minichiello L: Distinct requirements for TrkB and TrkC signaling in target innervation by sensory neurons. Genes Dev 2002, 16:633-645

9. Maina F, Pante G, Helmbacher F, Andres R, Porthin A, Davies AM, Ponzetto C, Klein R: Coupling Met to specific pathways results in distinct developmental outcomes. Mol Cell 2001, 7:1293-1306.

10. Encinas M, Tansey MG, Tsui-Pierchala BA, Comella JX, Milbrandt J, Johnson EM Jr: c-Src is required for glial cell line-derived neurotrophic factor (GDNF) family ligand-mediated neuronal survival via a phosphatidylinositol-3 kinase (PI-3 K)-dependent pathway. J Neurosci 2001, 21:1464-1472.

11. Tallquist MD, Klinghoffer RA, Heuchel R, Mueting-Nelsen PF, Corrin PD,

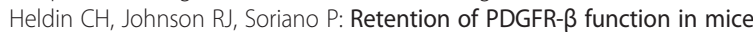
in the absence of phosphatidylinositol 3'-kinase and phospholipase $C_{\gamma}$ signaling pathways. Genes Dev 2000, 14:3179-3190.

12. Maina F, Hilton MC, Ponzetto C, Davies AM, Klein R: Met receptor signaling is required for sensory nerve development and HGF promotes axonal growth and survival of sensory neurons. Genes Dev 1997, 11:3341-3350.

13. Brand-Saberi B, Muller TS, Wilting J, Christ B, Birchmeier C: Scatter factor/ hepatocyte growth factor (SF/HGF) induces emigration of myogenic cells at interlimb level in vivo. Dev Biol 1996, 179:303-308.

14. Dietrich S, Abou-Rebyeh F, Brohmann H, Bladt F, Sonnenberg-Riethmacher E, Yamaai T, Lumsden A, Brand-Saberi B, Birchmeier C: The role of SF/HGF and c-Met in the development of skeletal muscle. Development 1999, 126:1621-1629.

15. Maina F, Casagranda F, Audero E, Simeone A, Comoglio PM, Klein R, Ponzetto C: Uncoupling of Grb2 from the Met receptor in vivo reveals complex roles in muscle development. Cell 1996, 87:531-542.

16. Helmbacher F, Dessaud E, Arber S, de Lapeyriere O, Henderson CE, Klein R, Maina F: Met signaling is required for recruitment of motor neurons to PEA3-positive motor pools. Neuron 2003, 39:767-777.

17. Caton A, Hacker A, Naeem A, Livet J, Maina F, Bladt F, Klein R, Birchmeier C, Guthrie S: The branchial arches and HGF are growth-promoting and chemoattractant for cranial motor axons. Development 2000, 127:1751-1766.

18. Ebens A, Brose K, Leonardo ED, Hanson MG Jr, Bladt F, Birchmeier C, Barres BA, Tessier-Lavigne M: Hepatocyte growth factor/scatter factor is an axonal chemoattractant and a neurotrophic factor for spinal motor neurons. Neuron 1996, 17:1157-1172.

19. Yamamoto Y, Livet J, Pollock RA, Garces A, Arce V, De Lapeyriere O, Henderson CE: Hepatocyte growth factor (HGF/SF) is a muscle-derived survival factor for a subpopulation of embryonic motoneurons. Development 1997, 124:2903-2913.

20. Yamamoto $Y$, Henderson CE: Patterns of programmed cell death in populations of developing spinal motoneurons in chicken, mouse, and rat. Dev Biol 1999, 214:60-71.

21. Huh CG, Factor VM, Sanchez A, Uchida K, Conner EA, Thorgeirsson SS: Hepatocyte growth factor/c-met signaling pathway is required for efficient liver regeneration and repair. Proc Natl Acad Sci U S A 2004, 101:4477-4482.

22. Ponzetto C, Bardelli A, Zhen Z, Maina F, dalla Zonca P, Giordano S, Graziani A, Panayotou G, Comoglio PM: A multifunctional docking site mediates signaling and transformation by the hepatocyte growth factor/scatter factor receptor family. Cell 1994, 77:261-271.

23. Furlan A, Lamballe F, Stagni V, Hussain A, Richelme S, Prodosmo A, Moumen A, Brun C, Del Barco BI, Arthur JS, Koleske AJ, Nebreda AR, Barila D, Maina F: Met acts through Abl to regulate p53 transcriptional outcomes and cell survival in the developing liver. J Hepatol 2012, 57:1292-1298. 
24. Moumen A, Patane S, Porras A, Dono R, Maina F: Met acts on Mdm2 via mTOR to signal cell survival during development. Development 2007, 134:1443-1451.

25. Moumen A, leraci A, Patane S, Sole C, Comella JX, Dono R, Maina F: Met signals hepatocyte survival by preventing Fas-triggered FLIP degradation in a PI3k-Akt-dependent manner. Hepatology 2007, 45:1210-1217.

26. Segarra J, Balenci L, Drenth T, Maina F, Lamballe F: Combined signaling through ERK, $\mathrm{PI} 3 \mathrm{~K} / \mathrm{AKT}$, and $\mathrm{RAC} 1 / \mathrm{p} 38$ is required for met-triggered cortical neuron migration. J Biol Chem 2006, 281:4771-4778.

27. Gu WX, Kania A: Examining the combinatorial model of motor neuron survival by expression profiling of trophic factors and their receptors in the embryonic Gallus gallus. Dev Dyn 2010, 239:965-979.

28. Sonnenberg E, Meyer D, Weidner KM, Birchmeier C: Scatter factor/ hepatocyte growth factor and its receptor, the c-met tyrosine kinase, can mediate a signal exchange between mesenchyme and epithelia during mouse development. J Cell Biol 1993, 123:223-235.

29. Helmbacher F, Schneider-Maunoury S, Topilko P, Tiret L, Charnay P: Targeting of the EphA4 tyrosine kinase receptor affects dorsal/ventral pathfinding of limb motor axons. Development 2000, 127:3313-3324.

30. Messina G, Cossu G: The origin of embryonic and fetal myoblasts: a role of Pax3 and Pax7. Genes Dev 2009, 23:902-905.

31. Haase G, Dessaud E, Garces A, de Bovis B, Birling M, Filippi P, Schmalbruch $\mathrm{H}$, Arber S, de Lapeyriere O: GDNF acts through PEA3 to regulate cell body positioning and muscle innervation of specific motor neuron pools. Neuron 2002, 35:893-905.

32. Livet J, Sigrist M, Stroebel S, De Paola V, Price SR, Henderson CE, Jessell TM, Arber S: ETS gene Pea3 controls the central position and terminal arborization of specific motor neuron pools. Neuron 2002, 35:877-892.

33. Novak KD, Prevette D, Wang S, Gould TW, Oppenheim RW: Hepatocyte growth factor/scatter factor is a neurotrophic survival factor for lumbar but not for other somatic motoneurons in the chick embryo. J Neurosci 2000, 20:326-337.

34. Arce V, Garces A, de Bovis B, Filippi P, Henderson C, Pettmann B, de Lapeyriere O: Cardiotrophin-1 requires LIFRbeta to promote survival of mouse motoneurons purified by a novel technique. J Neurosci Res 1999, 55:119-126.

35. Dolcet X, Soler RM, Gould TW, Egea J, Oppenheim RW, Comella JX: Cytokines promote motoneuron survival through the Janus kinasedependent activation of the phosphatidylinositol 3-kinase pathway. Mol Cell Neurosci 2001, 18:619-631.

36. Datta SR, Brunet A, Greenberg ME: Cellular survival: a play in three Akts. Genes Dev 1999, 13:2905-2927.

37. Uda S, Saito TH, Kudo T, Kokaji T, Tsuchiya T, Kubota H, Komori Y, Ozaki Y, Kuroda S: Robustness and compensation of information transmission of signaling pathways. Science 2013, 341:558-561.

38. Citri A, Yarden Y: EGF-ERBB signalling: towards the systems level. Nat Rev Mol Cell Biol 2006, 7:505-516.

39. Stelling J, Sauer U, Szallasi Z, Doyle FJ 3rd, Doyle J: Robustness of cellular functions. Cell 2004, 118:675-685.

40. Wagner JP, Wolf-Yadlin A, Sevecka M, Grenier JK, Root DE, Lauffenburger DA, MacBeath G: Receptor tyrosine kinases fall into distinct classes based on their inferred signaling networks. Sci Signal 2013, 6:ra58.

41. Ciriello G, Cerami E, Sander C, Schultz N: Mutual exclusivity analysis identifies oncogenic network modules. Genome Res 2012, 22:398-406.

42. Cerami E, Demir E, Schultz N, Taylor BS, Sander C: Automated network analysis identifies core pathways in glioblastoma. PLoS One 2010, 5:e8918.

43. Matsuoka H, Sisson TH, Nishiuma T, Simon RH: Plasminogen-mediated activation and release of hepatocyte growth factor from extracellular matrix. Am J Respir Cell Mol Biol 2006, 35:705-713.

44. Komada M, Hatsuzawa K, Shibamoto S, Ito F, Nakayama K, Kitamura N: Proteolytic processing of the hepatocyte growth factor/scatter factor receptor by furin. FEBS Lett 1993, 328:25-29.

45. Naldini L, Tamagnone L, Vigna E, Sachs M, Hartmann G, Birchmeier W, Daikuhara Y, Tsubouchi H, Blasi F, Comoglio PM: Extracellular proteolytic cleavage by urokinase is required for activation of hepatocyte growth factor/scatter factor. EMBO J 1992, 11:4825-4833.

46. Kao TJ, Palmesino E, Kania A: SRC family kinases are required for limb trajectory selection by spinal motor axons. J Neurosci 2009, 29:5690-5700.

47. Bonanomi D, Chivatakarn O, Bai G, Abdesselem H, Lettieri K, Marquardt T, Pierchala BA, Pfaff SL: Ret is a multifunctional coreceptor that integrates diffusible- and contact-axon guidance signals. Cell 2012, 148:568-582.
48. Bonanomi D, Pfaff SL: Motor axon pathfinding. Cold Spring Harb Perspect Biol 2010, 2:a001735.

49. Kramer ER, Knott L, Su F, Dessaud E, Krull CE, Helmbacher F, Klein R: Cooperation between GDNF/Ret and ephrinA/EphA4 signals for motor-axon pathway selection in the limb. Neuron 2006, 50:35-47

50. O'Donnell MP, Bashaw GJ: Src inhibits midline axon crossing independent of Frazzled/Deleted in Colorectal Carcinoma (DCC) receptor tyrosine phosphorylation. J Neurosci 2013, 33:305-314.

51. Zimmer M, Palmer A, Kohler J, Klein R: EphB-ephrinB bi-directional endocytosis terminates adhesion allowing contact mediated repulsion. Nat Cell Biol 2003, 5:869-878.

52. Palmer A, Zimmer M, Erdmann KS, Eulenburg V, Porthin A, Heumann R, Deutsch $U$, Klein R: EphrinB phosphorylation and reverse signaling: regulation by Src kinases and PTP-BL phosphatase. Mol Cell 2002, 9:725-737.

53. Liu G, Beggs H, Jurgensen C, Park HT, Tang H, Gorski J, Jones KR, Reichardt $L F$, Wu J, Rao Y: Netrin requires focal adhesion kinase and Src family kinases for axon outgrowth and attraction. Nat Neurosci 2004 7:1222-1232.

54. Wolf AM, Lyuksyutova Al, Fenstermaker AG, Shafer B, Lo CG, Zou Y: Phosphatidylinositol-3-kinase-atypical protein kinase $C$ signaling is required for Wnt attraction and anterior-posterior axon guidance. J Neurosci 2008, 28:3456-3467.

55. Charoy C, Nawabi H, Reynaud F, Derrington E, Bozon M, Wright K, Falk J, Helmbacher F, Kindbeiter K, Castellani V: gdnf activates midline repulsion by semaphorin3B via NCAM during commissural axon guidance. Neuron 2012, 75:1051-1066.

56. Chauvet S, Cohen S, Yoshida Y, Fekrane L, Livet J, Gayet O, Segu L, Buhot MC, Jessell TM, Henderson CE, Mann F: Gating of Sema3E/PlexinD1 signaling by neuropilin-1 switches axonal repulsion to attraction during brain development. Neuron 2007, 56:807-822

57. Hopker VH, Shewan D, Tessier-Lavigne M, Poo M, Holt C: Growth-cone attraction to netrin-1 is converted to repulsion by laminin-1. Nature 1999, 401:69-73.

58. Tsang M, Dawid IB: Promotion and attenuation of FGF signaling through the Ras-MAPK pathway. Sci STKE 2004, 2004:e17.

59. Brent $A E$, Tabin CJ: FGF acts directly on the somitic tendon progenitors through the Ets transcription factors Pea3 and Erm to regulate scleraxis expression. Development 2004, 131:3885-3896.

60. O'Hagan RC, Tozer RG, Symons M, McCormick F, Hassell JA: The activity of the Ets transcription factor PEA3 is regulated by two distinct MAPK cascades. Oncogene 1996, 13:1323-1333.

61. Janknecht $R$, Monte $D$, Baert JL, de Launoit $Y$ : The ETS-related transcription factor ERM is a nuclear target of signaling cascades involving MAPK and PKA. Oncogene 1996, 13:1745-1754.

62. Janknecht R: Analysis of the ERK-stimulated ETS transcription factor ER81. Mol Cell Biol 1996, 16:1550-1556.

63. Fontanet $\mathrm{P}$, Irala $\mathrm{D}$, Alsina $\mathrm{FC}$, Paratcha $\mathrm{G}$, Ledda F: Pea3 transcription factor family members Etv4 and Etv5 mediate retrograde signaling and axonal growth of DRG sensory neurons in response to NGF. J Neurosci 2013, 33:15940-15951.

64. Vincenti MP, Schroen DJ, Coon Cl, Brinckerhoff CE: v-src activation of the collagenase-1 (matrix metalloproteinase-1) promoter through PEA3 and STAT: requirement of extracellular signal-regulated kinases and inhibition by retinoic acid receptors. Mol Carcinog 1998, 21:194-204.

65. Chaar ZY, Hastings L, Sriram R, McKay M, Antonova L, Hassell JA, Sabourin LA: Impaired c-src activation and motility defects in PEA3-null fibroblasts. Biochim Biophys Acta 1823, 2012:2237-2242.

66. Duronio V: The life of a cell: apoptosis regulation by the PI3K/PKB pathway. Biochem J 2008, 415:333-344.

67. Brunet A, Datta SR, Greenberg ME: Transcription-dependent and -independent control of neuronal survival by the PI3K-Akt signaling pathway. Curr Opin Neurobiol 2001, 11:297-305.

68. Engelman JA: Targeting PI3K signalling in cancer: opportunities, challenges and limitations. Nat Rev Cancer 2009, 9:550-562.

69. Encinas M, Crowder RJ, Milbrandt J, Johnson EM Jr: Tyrosine 981, a nove ret autophosphorylation site, binds c-Src to mediate neuronal survival. J Biol Chem 2004, 279:18262-18269.

70. Xing L, Venegas AM, Chen A, Garrett-Beal L, Boyce BF, Varmus HE, Schwartzberg PL: Genetic evidence for a role for Src family kinases in TNF family receptor signaling and cell survival. Genes Dev 2001, 15:241-253. 
71. Egea J, Espinet C, Soler RM, Dolcet X, Yuste VJ, Encinas M, Iglesias M, Rocamora N, Comella JX: Neuronal survival induced by neurotrophins requires calmodulin. J Cell Biol 2001, 154:585-597.

72. GenePaint database. http://www.genepaint.org/Frameset.html.

73. Genestine M, Caricati E, Fico A, Richelme S, Hassani H, Sunyach C, Lamballe F, Panzica GC, Pettmann B, Helmbacher F, Raoul C, Maina F, Dono R: Enhanced neuronal Met signalling levels in ALS mice delay disease onset. Cell Death Dis 2011, 2:e130.

74. Garces A, Haase G, Airaksinen MS, Livet J, Filippi P, de Lapeyriere O: GFRa1 is required for development of distinct subpopulations of motoneuron. J Neurosci 2000, 20:4992-5000.

75. Sundararajan S, Wakamiya M, Behringer RR, Rivera-Perez JA: A fast and sensitive alternative for beta-galactosidase detection in mouse embryos. Development 2012, 139:4484-4490.

doi:10.1186/s12915-014-0056-6

Cite this article as: Caruso et al:: Plasticity versus specificity in RTK

signalling modalities for distinct biological outcomes in motor neurons. BMC Biology 2014 12:56.

\section{Submit your next manuscript to BioMed Central and take full advantage of:}

- Convenient online submission

- Thorough peer review

- No space constraints or color figure charges

- Immediate publication on acceptance

- Inclusion in PubMed, CAS, Scopus and Google Scholar

- Research which is freely available for redistribution 